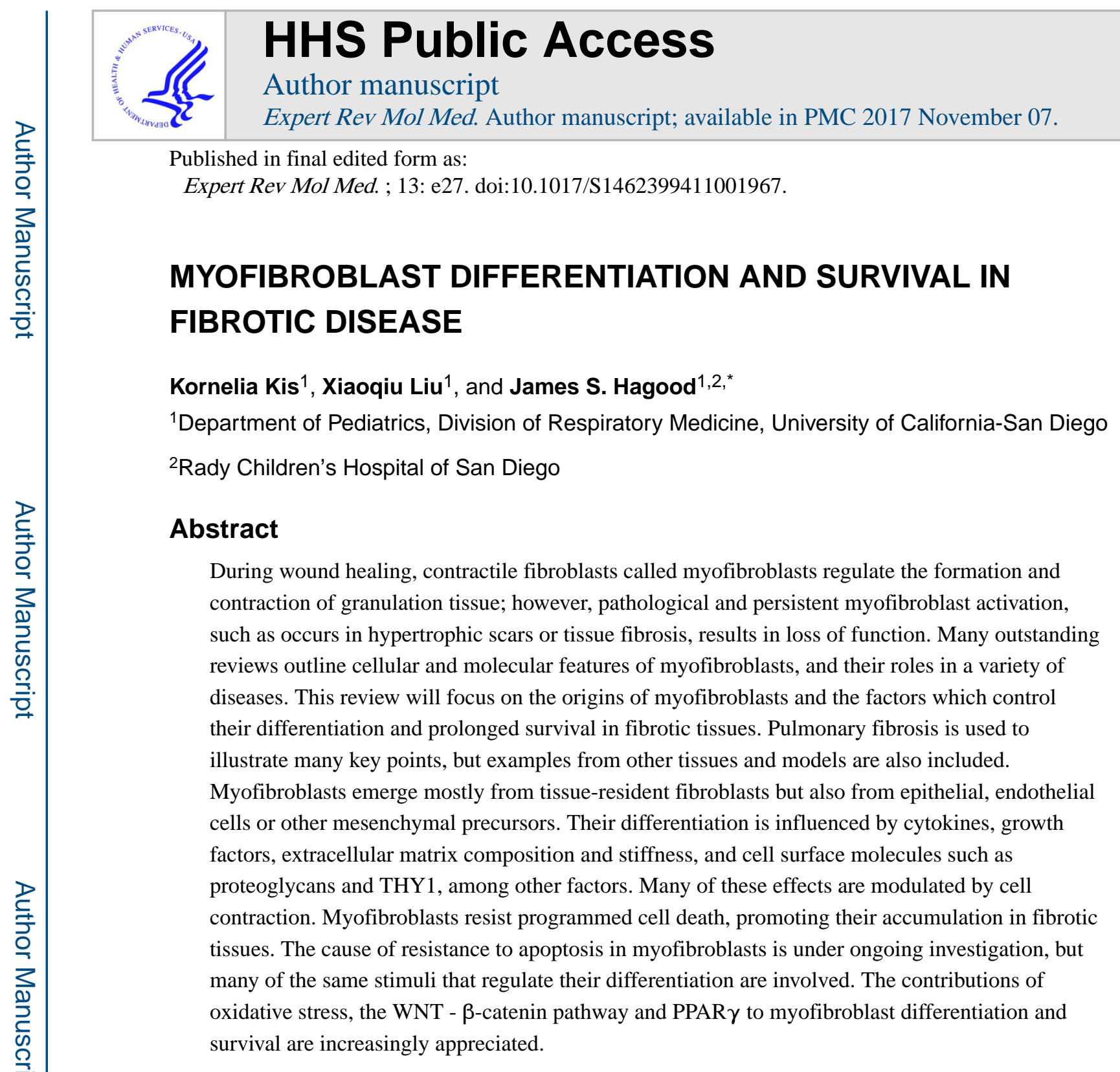

Keywords

myofibroblast differentiation; apoptosis; TGFB; alpha smooth muscle actin; fibrosis

\title{
Introduction
}

There are a number of diseases characterized by the accumulation of excessive or inappropriate scar tissue resulting in organ dysfunction. Notable examples include idiopathic pulmonary fibrosis, liver fibrosis, kidney fibrosis, and systemic sclerosis/scleroderma. Many of these occur in tissues with epithelial/mesenchymal/vascular structures in close opposition. In a broader context, many other disorders with prominent tissue remodeling, such as cardiac failure, atherosclerosis, and asthma have a significant fibrotic component. Although almost every cell type has been implicated in the pathogenesis of fibrosis, fibroblasts, as the

*corresponding author: University of California-San Diego, 9500 Gilman Dr. MC 0731, San Diego, CA 92093-0731, jhagood@ucsd.edu. 
mediators of fibrillar collagen deposition, are the cellular sine qua non. Fibroblasts are somewhat ill-defined. They are found in most tissues, especially ones with prominent epithelial and microvascular components (e.g., skin, lung, liver, kidney). Fibroblasts are identified primarily by their location and morphology. In tissue culture, they are often characterized by their appearance and the absence of specific markers. Fibroblasts are important in normal development, tissue homeostasis, and wound healing. Injury and repair of most tissues begins with epithelial and/or endothelial damage, resulting in exudation of blood or platelet-rich plasma into extravascular spaces, leading to formation of a fibrinous clot (provisional matrix) (Ref. 1). Fibroblasts migrate into the provisional matrix, proliferate, and produce de novo extracellular matrix (ECM) components, such as fibronectin and collagen, creating a fibroblast-populated granulation tissue. Simultaneously, fibroblasts acquire a myofibroblastic phenotype. Because myofibroblasts actively elaborate and remodel abundant ECM, and because persistence and activation of myofibroblasts is seen in abnormal scar tissue, myofibroblasts are thought to be the end effector cell and "final common pathway" for most fibrotic diseases. In the first part of this review, factors promoting myofibroblastic differentiation will be considered. The principal mediators affecting myofibroblast differentiation are listed in Table 1, and the main signaling pathways involved are depicted in Figure 2. In the latter half of this review, factors affecting the prolonged survival of myofibroblasts in scar tissue will be discussed.

\section{Origin of myofibroblasts}

Specialized contractile fibroblasts called myofibroblasts emerge following tissue injury; their role in normal wound healing has been well-described (Ref. 2). Fibroblasts are the principal effector cells during fibrosis (scarring) in most tissues, as evidenced both by their persistence and excessive matrix deposition (mostly collagen I, III and fibronectin) (Ref. 3). Although tissue fibrosis (e.g. idiopathic pulmonary fibrosis, IPF, a chronic disease with progressive scarring of the lung parenchyma) was originally thought to be mediated exclusively by resident mesenchymal cells that proliferated and synthesized excessive amount of extracellular matrix (Ref. 2, 4), it has recently been demonstrated in many tissues that myofibroblasts can also be derived from epithelial to mesenchymal transition (EMT), endothelial-mesenchymal transition (EndMT), and from bone marrow-derived mesenchymal precursors (fibrocytes or mesenchymal stem cells). Whether myofibroblasts derived from different cellular origins have distinct roles in the injury-repair process, and whether they take part in the normal repair response as opposed to fibrosis, have yet to be elucidated. The possible cellular origins of myofibroblasts are depicted in Figure 1.

\section{Epithelial-mesenchymal transition (EMT)}

EMT plays a crucial role in the differentiation of multiple tissues and organs and in tissue repair, and can also contribute to organ fibrosis and promote carcinoma progression via the local formation of interstitial myofibroblasts from organ epithelium (Ref. 5). Mediators triggering EMT include different members of the transforming growth factor $\beta$ (TGFB) superfamily, wingless-type MMTV integration site family members (WNTs), Notch, epidermal growth factor (EGF), hepatocyte growth factor (HGF), fibroblast growth factor (FGF), hypoxia inducible factor (HIF) and many others (Ref. 5, 6). A wide array of 
transcription factors such as SNAI1 and TWIST1 are involved in regulating EMT (Ref. 7). Fibroblasts expressing fibroblast-specific protein-1 (FSP1, also called S100A4) can arise in large numbers by local EMT during renal fibrogenesis (Ref. 8). Although FSP1 is often used as a fibroblast marker, it has also been shown to be a marker of a specific subset of inflammatory macrophages in liver injury, fibrosis and cancer (Ref. 9). Bone morphogenic protein 7 (BMP7), a member of the TGFB superfamily that is known to antagonize TGFB signaling, has been demonstrated to inhibit progression of fibrosis and to abolish EMT- or EndMT-derived fibroblasts in liver (Ref. 10), cardiac (Ref. 11) and kidney fibrosis (Ref. 12). In the presence of active TGFB1, a $3 \beta 1$ integrin mediates tyrosine phosphorylation of $\beta$ catenin at Y654 and formation of pY- $\beta$-catenin/pSMAD2 transcriptional complexes in alveolar epithelial cells, promoting EMT and contributing to lung fibrosis (Ref. 13). Human bronchial epithelial cells are also able to undergo EMT in vitro upon TGFB stimulation via a primarily SMAD2/3 dependent mechanism (Ref. 14). However, other groups have found no evidence of EMT in liver (Ref. 15) or kidney (Ref. 16) fibrosis. Despite many recent studies defining mechanisms of EMT, its exact role in vivo in many diseases and models remains in question (Ref. 17).

\section{Endothelial-mesenchymal transition (EndMT)}

EndMT plays an important role during cardiovascular development and in various vascular pathologies. There are many similarities between EMT and EndMT regarding the loss of expression of cell-specific markers and increased expression of mesenchymal markers such as alpha 2 smooth muscle actin (ACTA2), fibronectin, FSP1 and collagen I and III (reviewed in (Ref. 18, 19, 20)). Furthermore, EndMT is also induced by TGFB1 in a SMAD2/3dependent manner and can be reversed by bone morphogenetic protein 7 (BMP7) during cardiac fibrosis (Ref. 11). FSP1 expression in human umbilical vein endothelial cells (HUVECs) induced by high glucose levels is inhibited by endothelin 1 (ET1, EDN1) siRNA along with decreased phosphorylation of SMAD3 and AKT, decreased expression of TGFB and SNAI1 and decreased motility of these cells (Ref. 21) suggesting a role of ET1 in EndMT. EndMT has been also demonstrated in bleomycin-induced lung fibrosis in lacZexpressing TIE2-cre transgenic mice (Ref. 22). The contribution of EndMT to vascular remodeling (Ref. 23), cancer progression (Ref. 20) and diabetic renal fibrosis (Ref. 18) has been reviewed elsewhere.

\section{Fibrocytes}

A population of circulating cells with fibroblast properties, called fibrocytes, was first described in 1994. These cells specifically enter sites of tissue injury and express collagen I, vimentin, fibronectin, CD11b, beta 2 integrin (ITGB2), CD34, protein tyrosine phosphatase, receptor type, C (PTPRC), but are negative for esterase, CD14, CD25, and CD54 expression. Although in a sex-mismatched bone marrow chimeric mouse model the fibrocytes present in wound chambers did not arise from the transferred bone marrow, others have demonstrated bone marrow origin of lesional cells during lung fibrosis (Ref. 24) and renal fibrosis (Ref. 8), and their numbers in blood have been demonstrated to correlate with disease activity of IPF (Ref. 25). Several chemoattractants, such as CCL2(Ref. 26), stromal cell-derived factor 1 (SDF-1a, also called CXCL12) (Ref. 24, 27, 28, 29), platelet-derived growth factor beta (PDGFB) (Ref. 28), and secondary lymphoid tissue chemokine (SLC, also called CCL21) 
(Ref. 24, 30) have been shown to play a role in fibrocyte migration in vitro or in vivo during lung fibrosis indicating that circulating fibrocytes may contribute to the expansion of the fibroblast/ myofibroblast population in IPF (Ref. 31). An elegant lineage tracing study in the bleomycin model has demonstrated that in the active fibrotic stage two weeks after injury, one-third of FSP1+ cells were of epithelial origin and one-fifth of bone marrow origin, but that neither of these contributed significantly to lesional myofibroblasts (Ref. 17). Thus it is possible that EMT, EndMT, and fibrocytes or other bone-marrow derived precursors may have more important roles in normal repair than in fibrosis.

\section{Mesenchymal stem cells (MSC)}

Although exogenous MSCs can ameliorate fibrosis (Ref. 32), MSCs from tracheal aspirates of premature infants, but not bone marrow-derived MSCs, also have the capacity to differentiate into myofibroblasts in response to TGFB1 (Ref. 33). Bone marrow-derived MSCs require an additional profibrotic signal such as CTGF to complete their differentiation from fibroblastic to myofibroblastic phenotype (Ref. 34).

\section{Cytokines and growth factors}

Sources for profibrotic cytokines in the lungs include macrophages and other inflammatory cells, epithelial and mesenchymal cells. TGFB1, tumor necrosis factor (TNF-a), platelet derived growth factor (PDGF), connective tissue growth factor (CTGF), interleukin 1, beta (IL-1B), IL-6 and IL-4 have well-established roles in myofibroblast differentiation (Ref. 35, 36, 37). Regulators of these key players and roles of other cytokines are under ongoing investigation. Understanding networks of cytokine and growth factor interactions is probably more important than the roles of individual mediators. TGFB1 has a central role, not only in inducing myofibroblastic differentiation and fibrosis, but also in the differentiation of regulatory $\mathrm{T}$ cells that suppress lymphocyte activation. However, in the presence of IL-6 (for mouse cells) or IL-21 (for human cells), TGFB1 facilitates the generation of proinflammatory, IL-17-producing TH17 T helper lymphocytes (Ref. 38). Bleomycin- and IL-1B-induced IL-17A production and lung fibrosis is TGFB-dependent, suggesting cooperative roles for IL-17A and TGFB in the development of fibrosis (Ref. 39). IL-17A and IL-17F stimulate inflammatory responses in human colonic subepithelial myofibroblasts that contribute to the pathogenesis of inflammatory bowel disease (Ref. 40). IL-6 is produced by IL-1A-stimulated human cardiac myofibroblasts (Ref. 41) and during acute exacerbations of IPF (Ref. 42). The effect of IL-4 is somewhat contradictory: based on intratracheal bleomycin administration to IL-4 -/- or IL-4 +/+ mice, IL-4 appears to have an early anti-inflammatory/immunosuppressive role, however, during later stages of fibrosis, IL-4 seems to play a profibrotic role (Ref. 43). THY1 (-), but not THY1 (+), rat lung fibroblasts respond to PDGFBB, IL-1B, IL-4, or bleomycin treatment with increased TGFB activity, SMAD3 phosphorylation, and expression of ACTA2 and fibronectin (Ref. 44). TGFB activation by IL-4 and bleomycin is mediated by thrombospondin 1 (TSP-1), whereas the effects of IL-1B require the activation of plasmin and matrix metallopeptidases (MMPs) (Ref. 44). The mutant form of TGFB3 with improved bioavailability and activity reduces fibroblast/myofibroblast transdifferentiation and re-epithelialization density in a mouse skin wounding model (Ref. 45). TNF- $a$ antagonizes TGFB1-mediated human pulmonary 
myofibroblast differentiation via ERK1/2-dependent induction of the early growth response 1 (EGR1) (Ref. 46).

\section{Myogenic transcription factors}

Myogenesis, which is required for development and regeneration of skeletal muscle, is regulated by myogenic regulatory factors including MYOD1 and myogenin. Mitogenactivated protein kinase kinase kinase 7 (MAP3K7) has been shown to induce myogenic differentiation through the activation of p38 MAPK and AKT kinase in mouse myoblasts and embryonic fibroblasts, while the RAS/RAF/MEK/ERK pathway was inhibitory (Ref. 47). FIZZ1 (found in inflammatory zone; also known as resistin-like alpha), is increased markedly in fibrotic lungs and induces myofibroblast differentiation (Ref. 48). Similarly, Yin Yang 1 (YY1) is found in fibrotic lung and potently induces fibroblast ACTA2 expression (Ref. 49).

\section{THY1}

A distinct feature of IPF is the development of fibroblastic foci, which represent areas of active fibrosis and correlate most clearly with poor outcome. Fibroblasts within fibroblastic foci of usual interstitial pneumonia (UIP)/IPF patients express ACTA2, but show absent expression of THY1 (Ref. 50, 51). Intratracheal bleomycin induces more severe lung fibrosis in Thy1 knockout mice demonstrated by histological evaluation and TGFB activation (Ref. 50), indicating a lung fibrosis-suppressing function of THY1. Confirming the in vivo results in lung fibrosis, THY1 (-) rat lung fibroblasts have significantly higher myofibroblast and myogenic regulatory factor gene and protein expression, cause significantly greater collagen contraction and are more resistant to apoptosis compared to THY1 (+) cells (Ref. 52). Most of the myofibroblastic features of THY1 (-) lung fibroblasts are reversible upon reexpression of THY1 (Ref. 52, 53), indicating an important mechanistic role. Indeed, this has been confirmed by demonstrating that THY1 inhibits activation of latent TGFB and myofibroblastic differentiation in lung fibroblasts (Ref. 44, 54). Unlike lung fibroblasts, only THY1 (+) TGFB-treated human myometrial or orbital fibroblasts expressed ACTA2 while only THY1 (-) orbital fibroblasts differentiated to lipofibroblasts (Ref. 55), possibly due to paracrine anti-adipogenic factors secreted by THY1 expressing cells (Ref. 56). This seemingly contradicting modulation of fibrosis by THY1 may be due to differences in local tissue environment (e.g. ECM and integrin expression). The role of TGFB and other fibrogenic factors in myogenic differentiation of fibroblasts (i.e. expression of MYOD and myogenin together with ACTA2, sarcomeric myosin, myocardin and desmin) was confirmed in THY1(-) fibroblasts (Ref. 52).

\section{Extracellular matrix, physical force and cell contractility}

In early granulation tissue, the mechanical tension of the open wound gives the initiation signal for the differentiation of proto-myofibroblasts (Ref. 57, 58), which show the morphological characteristics of myofibroblasts, such as stress fibers, but do not express ACTA2. Lack of mechanical load on fibroblasts in a collagen matrix (similar to the environment of an early wound) produces an "inflammatory" phenotype that is characterized by high expression of inflammatory mediators and proteases along with low proliferation (Ref. 57). Further differentiation to mature, ACTA2-expressing myofibroblasts is driven by 
high tissue stiffness, the presence of TGFB1 and the ED-A splice variant of fibronectin in later stages of wound healing. Mechanical load induces an "activated" proliferating fibroblast phenotype that synthesizes abundant ECM and protease inhibitors as well as fibrogenic mediators. Integrins connect the cytoskeleton to the ECM and are able to transmit mechanical signals in both directions across the plasma membrane (Ref. 59). Release of active TGFB from the latent TGFB complex has been shown to be mediated by RhoA activation via the protease-activated receptor 1 (PAR-1) in an av $\beta 6$ integrin-dependent manner in epithelial cells (Ref. 60), probably as a result of cytoskeletal changes, in addition to TSP-1- and CD36-mediated protease activity (Ref. 61). This observation is supported by results showing that myofibroblast contraction directly releases active TGFB1 from the mechanoresistant ECM via integrins (Ref. 62). THY1 has been shown to bind integrin av $\beta 5$ of rat lung fibroblasts and to inhibit contraction-induced latent TGFB1 activation and TGFB1-dependent lung myofibroblast differentiation, presumably by interfering the binding of latent TGFB with integrin av $\beta 5$ (Ref. 54). The functional integrin-binding motif (RLD) of THY1 is necessary for contraction-induced lung myofibroblast differentiation (Ref. 54). Furthermore, THY1 knockout mice with exacerbated lung fibrosis and increased active TGFB level have increased LTBP4 expression as compared with wild-type mice (Ref. 63) suggesting another mechanism by which THY1 is able to reduce the release of active TGFB. Integrins regulate cell spreading and migration through activation of the Rho family of small GTPases such as RhoA, CDC42 and RAC (Ref. 64). RAC1 becomes activated during differentiation of quiescent hepatic stellate cells (HSCs) into myofibroblastic cells (Ref. 65). A constitutively active form of RAC1 was found to generate enhanced Hedgehog $(\mathrm{HH})$ signaling, with down-regulation of HHIP and increased expression of SHH (HH ligand) and GLI2, both in vitro in myofibroblastic HSCs and in vivo in a bile duct ligation-induced mouse liver fibrosis model (Ref. 65). The crosstalk between specific integrins and TGFB signaling is reviewed elsewhere (Ref. 66). Actin assembly is regulated by formin proteins, such as the mammalian Diaphanous-regulated formin (DIA) that becomes activated after interaction with Rho family of small GTPases. DIA has been shown to mediate forceinduced myofibroblast differentiation by increasing translocation of myocardin-related transcription factor-A (MRTF-A, also called MKL1) and activation of serum response factor (SRF), activators of ACTA2 transcription (Ref. 67). Changes in intracellular free cytoplasmic $\mathrm{Ca}^{2+}$ concentration contribute to myofibroblast differentiation by influencing cell contractility and mechanical communication between cells (reviewed in (Ref. 68)).

Most matrix proteins (fibronectin, vitronectin, TSP-1, laminin and collagens) contain binding domains for members of the integrin family as well as heparin-binding domains (HBDs) that mediate interactions with cell surface proteoglycans such as syndecans (Ref. $69,70)$. Syndecans can regulate microfilament assembly and cell shape and influence small GTPase activation. CTGF (CCN2 ) is required for syndecan 4 (SDC4) and TSP-1 expression and loss of syndecan 4 expression is responsible for at least part of the CTGF -/- phenotype (Ref. 71). CTGF -/- mouse embyonic fibroblasts show a disorganized actin network, impaired migration and less contractile force (Ref. 71). The role of syndecan 4 in lung fibrosis has been demonstrated in that administration of recombinant CXCL10 protein, an antifibrotic chemokine that directly interacts with syndecan 4, inhibited fibrosis in WT mice, but not in SDC4 -/- mice (Ref. 72). Beside the THY1-integrin-binding motif (RLD), the 
heparin-binding domain of THY1, via interaction with syndecan 4, is also required to stimulate focal adhesion formation by stimulating RhoA via protein kinase $\mathrm{C}$, alpha (PRKCA) activation (Ref. 73). Therefore, the extracellular matrix plays an important role in the process of integrin- and THY1-mediated TGFB release and influences myofibroblast differentiation through signaling pathways such as the HH signaling and small GTPase activation by means of integrins, cell surface proteoglycans and THY1.

\section{Oxidative stress, cell metabolism}

NADPH oxidases (NOX) are multisubunit enzymes that generate superoxide by transferring electrons from NADPH to molecular oxygen. The production of reactive oxygen species (ROS) by NOX enzymes is important during phagocytic burst to kill pathogens and also for a variety of essential biological processes including developmental and differentiation processes, extracellular matrix cross-linking, hormone biosynthesis, cellular senescence, apoptosis, responses to oxygenation (oxygen sensing), and cellular signaling responses to growth factors, hormones, and cytokines (Ref. 74). The presence of an oxidant-antioxidant imbalance in IPF is indicated by increased oxidative processes and altered levels of antioxidants in the lungs of affected patients (Ref. 75). NOX4 mRNA and protein expression has been found to be elevated in TGFB1-treated human lung fibroblasts (Ref. 76), cardiac fibroblasts (Ref. 77) and in IPF lungs compared to controls (Ref. 78). ROS inhibitors decrease TGFB1-induced ACTA2 expression; furthermore, anti-NOX4 siRNA attenuates lung fibrosis (Ref. 76) and decreases TGFB1-induced SMAD2/3 phosphorylation in human cardiac fibroblasts (Ref. 77) and in fibroblasts from control and IPF lungs (Ref. 78) supporting the importance of NOX4 in myofibroblast differentiation. Oxidative stress can be stimulated by JUND inactivation or by treatment with desferrioxamine (DFO), which mimics hypoxia. The resulting accumulation of HIF-1a and phosphorylation of ERK1/2 leads to the activation of CXCL12/CXCR4 that converts fibroblasts into myofibroblasts in a carcinogenesis model (Ref. 79). Furthermore, overexpression of extracellular superoxide dismutase is able to attenuate bleomycin-induced lung fibrosis and vascular remodeling and blocks the early upregulation of TGFB1 and EGR1 (Ref. 80).

Beside myofibroblast differentiation, fibroblasts can also be differentiated into "lipofibroblasts" or even adipocytes. Adipogenesis is initiated by CCAAT/enhancer binding protein, beta (C/EBP- $\beta$ ) and $\gamma$ and driven by peroxisome proliferator-activated receptor gamma (PPAR $\gamma$ ) and plays an important role in conditions such as thyroid eye disease (TED) in which an autoimmune inflammatory response specifically targeted to the orbit results in remodeling and expansion of connective and adipose tissues, including proliferation and differentiation of fibroblasts to adipocytes (Ref. 56). THY1 can also influence lipofibroblast differentiation: only THY1 (-) orbital and myometrial fibroblasts differentiate to lipofibroblasts after treatment with PPAR $\gamma$ ligands (Ref. 55). The nuclear hormone receptor PPARs heterodimerize with retinoid X receptors (RXRs), thus forming a complex that regulates gene expression. PPAR $\gamma$ controls lipid and glucose metabolism and is also implicated in inflammation regulation. Primary human pulmonary fibroblasts express PPAR $\gamma$ and RXR proteins and are capable of PPAR $\gamma$-dependent gene transcription (Ref. 81, 82). The PPAR $\gamma$ agonist rosiglitazone (RSG) decreases FBS-induced proliferation, migration, and ACTA2 expression as well as FBS-, PDGFBB- or TGFB1-induced ERK 
phosphorylation of human fetal lung fibroblasts (Ref. 83). PPAR $\gamma$ agonists also inhibit TGFB-driven myofibroblast differentiation in vitro (Ref. 81, 82) by an AKT-dependent (Ref. 84) and SMAD2/3-, AP-1- and partially PPAR $\gamma$-independent fashion (Ref. 85), and inhibit in vivo bleomycin-induced lung fibrosis (Ref. 82). Heme oxygenase-1 (HO-1) is the inducible form of heme oxygenase that participates in the conversion of heme to equimolar concentrations of biliverdin, iron, and carbon monoxide and has antioxidant and antiinflammatory properties. Increased HO-1 expression by PPAR $\gamma$ ligands is also PPAR $\boldsymbol{\gamma}$ independent and can be reversed by $\mathrm{N}$-acetyl cysteine (NAC), a thiol antioxidant, but does not influence TGFB-induced ACTA2 expression (Ref. 86). TGFB can activate ERK1/2 and FAK/PI3K/AKT and inhibit PTEN in a SMAD-independent manner leading to myofibroblast differentiation and fibrosis (Ref. 84, 87). These effects can be partially reversed by resveratrol (Ref. 87) or PPAR $\boldsymbol{\gamma}$-ligands bearing and electrophilic center (Ref. 84).

\section{Other critical signaling pathways}

The WNT family proteins have been shown to play a role in organogenesis (e.g. kidney development), tissue homeostasis and tumor formation (Ref. 88). Activation of the WNT/ $\beta$ catenin signaling pathway leads to the nuclear translocation of $\beta$-catenin and complex formation with members of the T cell factor (TCF)/lymphoid enhancer factor-1 (LEF1) family of transcription factors. IPF fibroblasts constitutively express significantly more SPARC, a matricellular protein that modulates cell-matrix interactions, and nuclear $\beta$ catenin than control fibroblasts. SPARC mediates activation of $\beta$-catenin through activation of AKT and inhibition of glycogen synthase kinase 3 beta (GSK3B) (Ref. 89). In addition, SPARC regulates plasminogen activator inhibitor, type 1 (PAI-1, also called SERPINE1) expression in IPF fibroblasts (Ref. 89). After obstructive kidney injury, the expression of the majority of WNT family members is upregulated with accumulation of $\beta$-catenin in renal tubular epithelial cells of fibrotic kidney. Gene therapy with the WNT antagonist Dickkopf 1 inhibits myofibroblast activation and renal fibrosis (Ref. 90). ERK phosphorylation can also contribute to WNT pathway-activation by stabilizing c-Myc oncogene protein as shown in a murine neoplasia model (Ref. 91). TGFB can also influence the WNT pathway in the presence of a $3 \beta 1$ integrin by mediating the phosphorylation of $\beta$-catenin at position Y654 (in contrast to the Ser33, Ser37 and Thr41 phosphorylation by GSK3B) leading to EMT of alveolar epithelial cells and lung fibrosis (Ref. 13).

\section{Epigenetics, micro RNA, noncoding RNAs}

Epigenetic alterations result in heritable changes in gene function without changes in the DNA sequence, thus they offer an extra layer of transcriptional control regarding how, when and where genes are expressed (Ref. 92). Epigenetic regulation is important for the diversity of cell types arising during development, and it is also critical to maintain the stability and integrity of expressed gene profiles. DNA methylation and chromatin modifications have been extensively studied in cancer research. DNA methylation and histone modifications are closely related, though the critical initiating events in silencing remain to be clarified. DNA methylation tends to result in longer-term transcriptional silencing, while histone modifications may have shorter-term effects (Ref. 93). The post-translational modifications of histone proteins together with DNA methylation comprise the epigenome of a cell, which 
controls the access to the genes, and changes through development (Ref. 94). Other epigenetic mechanisms, such as microRNAs and chromatin structural alterations are also important. We have shown that although the $T H Y 1$ promoter region is usually demethylated in fibroblasts, hypermethylation can cause loss of THY1 expression in IPF (Ref. 53).

There is growing evidence for epigenetic alterations in fibrotic diseases. Methylation of Friend leukemia virus integration 1 (FLII) is associated with increased collagen expression in scleroderma fibroblasts (Ref. 95); histone deacetylase (HDAC) 4 is required for TGFBinduced myofibroblastic differentiation of skin fibroblasts (Ref. 96). Treatment of human lung fibroblasts with the pan-histone deacetylase (HDAC) inhibitor trichostatin A (TSA) blocks TGFB1-induced expression of ACTA2 and type 1 collagen, through an HDAC4dependent mechanism (Ref. 97). Epigenetic silencing of THY1 by DNA hypermethylation occurs specifically within fibroblastic foci in IPF (Ref. 53). Inhibition of DNA methyltranserase (DNMT) activity induces myofibroblastic differentiation of lung fibroblasts (Ref. 98). MicroRNAs are single-stranded RNA molecules of 21-23 nucleotides in length which can be complementary to multiple mRNAs and induce silencing of multiple transcripts. They have been found to regulate reprogramming of gene expression in several types of cancer and in fibrosis in other organs, such as the heart (Ref. 99). miR-21, which is found in lesional fibroblasts in lung fibrosis and increases expression of ACTA2 and fibronectin (Ref. 100), also mediates TGFB1-induced myofibroblastic differentiation in cancer stroma by targeting programmed cell death 4 (PDCD4), a negative regulator of several myofibroblastic genes (Ref. 101). The relationships among different epigenetic mechanisms can be complicated. Myofibroblastic differentiation of hepatic stellate cells involves an "epigenetic relay" system involving miR-132, methyl CpG binding protein 2 (MECP2), histone methylation, and PPAR $\gamma$ (Ref. 102).

\section{Section summary}

Tissue injury triggers the formation of myofibroblasts predominantly from fibroblasts, however, endothelial cells (EndMT), epithelial cells (EMT), mesenchymal stem cells and fibrocytes can also contribute to the myofibroblast pool (Figure 1). Besides the well-known functions of TGFB1, TNF-a, PDGF, CTGF, IL-1B, IL-6 and IL-4 in fibrosis, a cooperative role of IL-17A and TGFB1, as well as an antifibrotic effect of mutated TGFB3 have been suggested by recent studies. The extracellular matrix plays an important role in the process of integrin- and THY1-mediated TGFB activation and influences myofibroblast differentiation through signaling pathways such as HH signaling, small GTPase activation by means of integrins, cell surface proteoglycans and THY1. PPAR $\gamma$ ligands can antagonize several effects of TGFB1, while antioxidant strategies such as knocking down NOX4 decrease fibrosis by restoring the oxidant/antioxidant balance. Activation of WNT pathway and epigenetic changes such as methylation of THY1 and FLI1 promoters also contribute significantly to the fibrotic process.

\section{Myofibroblast survival/apoptosis}

Accumulated data support an important role for dysregulation of apoptotic cell death in the development of IPF, as well as fibrosis in most tissues (Ref. 103). Myofibroblasts, regardless 
of their origins (see above), are the key effector cells in fibrogenesis. In the process of normal wound healing, myofibroblasts undergo apoptosis and rapidly disappear; failure of apoptosis leads to myofibroblast expansion, excessive ECM production, persistent tissue contraction, and pathologic scar formation. In the lungs of IPF patients, a series of consistent findings support the correlation of dysregulated apoptosis with the pathogenesis of fibrosis (Ref. 104, 105, 106). In contrast, widespread epithelial apoptosis is observed, and epithelial apoptosis may be important in the initiation of fibrotic remodeling. The contrast of increased epithelial cell apoptosis with fibroblast resistance to apoptosis in IPF lung has been termed the "apoptosis paradox" (Ref. 104, 105, 107, 108, 109). Myofibroblast survival is a key determinant of whether fibrosis will progress or resolve (Ref. 110), and is due in part to enhanced responsiveness to growth factors and fibrotic cytokines in addition to resistance to apoptosis. Myofibroblast proliferation and survival require signals from the cellular and extracellular microenvironment, especially cell-cell and cell- matrix contacts that provide key signals for proliferation and survival. Modification of prosurvival signaling in fibroblasts by a protein kinase inhibitor (AG1879) protects against fibrotic tissue injury in bleomycin fibrosis model, indicating that targeting of prosurvival signaling pathways in fibroblasts/ myofibroblasts may provide a novel and effective anti-fibrotic therapy (Ref. 111, 112, 113). Recent studies have greatly improved our understanding of cellular and molecular factors regulating myofibroblast survival (Ref. 114, 115, 116, 117). The principal mediators affecting myofibroblast survival/apoptosis are listed in Table 2, and the main signaling pathways involved are depicted in Figure 3.

\section{Epithelial cell - fibroblast/myofibroblast interactions}

Products of epithelial cell injury may stimulate fibroblasts to produce ECM components such as collagen and fibronectin, leading to dysregulated repair of the epithelial/endothelial barrier (Ref. 118, 119, 120). IPF is thought to result from multiple cycles of epithelial cell injury and activation (Ref. 118, 121). Myofibroblasts from fibrotic lungs possess a cytotoxic phenotype that causes apoptosis of epithelial cells via the FAS/FasL pathway indicating that myofibroblast cytotoxicity may contribute to the absence of re-epithelialization, resulting in persistent lung fibrosis(Ref. 122). FasL-deficient myofibroblasts lose this myofibroblast cytotoxic phenotype both in vitro and in vivo. Blocking the FAS-FasL pathway may have a therapeutic potential in bleomycin-induced pulmonary fibrosis in mice(Ref. 123). However, in other studies the FAS/Fas-ligand system was shown not to be required for bleomycininduced pulmonary fibrosis in mice, suggesting that epithelial cell apoptosis after bleomycin exposure can be mediated by FAS-independent pathways as well(Ref. 124).

IPF lung fibroblasts are resistant to FasL-induced apoptosis(Ref. 125), but the mechanism is not completely understood. Altered expression of membrane-bound and soluble CD95/FAS contributes to the resistance of fibrotic lung fibroblasts to FasL induced apoptosis(Ref. 126). Prostaglandin $\left(\mathrm{PGE}_{2}\right)$ is an important antifibrotic lipid mediator(Ref. 127), the level of which is decreased in cultured lung fibroblasts isolated from IPF patients(Ref. 128). Prostaglandin E2 induces fibroblast apoptosis by modulating multiple survival pathways(Ref. 116). Exogenous $\mathrm{PGE}_{2}$ increases FasL-induced apoptosis in fibrotic lung fibroblasts compared with FasL alone. Conversely, in primary fibrotic lung type II alveolar epithelial cells (AEC), $\mathrm{PGE}_{2}$ protected against FasL-induced apoptosis, suggesting that 
diminished $\mathrm{PGE}_{2}$ contributes to the apoptosis paradox in IPF(Ref. 129). Administration of TGFB enhances Fas-mediated epithelial cell apoptosis and lung injury via caspase 3 activation in mice(Ref. 130). On the other hand TGFB1 inhibits FAS-mediated apoptosis in keloid-derived fibroblasts(Ref. 131).

\section{Pro- apoptotic and anti-apoptotic proteins}

Resistance of IPF fibroblasts to FAS mediated apoptosis has been associated with increased expression of X-linked inhibitor of apoptosis (XIAP) and CASP8 and FADD-like apoptosis inhibitor protein (FLIP) (Ref. 132). A more recent study showed that late-stage lesional fibroblasts from skin in patients with systemic sclerosis (SSc) were more resistant to FASinduced apoptosis than their non-lesional counterparts isolated from the same patient and correlated with an increase in the levels of the anti-apoptotic proteins cFLIPs and cIAP in lesional cells compared to non-lesional dermal fibroblasts, suggesting that cFLIPs and cIAP confer protection from Fas-induced apoptosis in SSc late-stage fibroblast populations(Ref. 103). Bcl-2 survival factors are critical for cardiac fibroblast resistance to apoptosis, and may explain the continued survival and accumulation of myofibroblasts in lung fibrosis (Ref. 133, 134). AEC from BID -/- mice are resistant to TGFB-induced cell death, suggesting that Bcl-2 family members are important regulators of fibrosis downstream of TGFB1 activation (Ref. 135).

\section{Prosurvival factors: growth factors and cytokines}

TGFB1 promotes an anti-apoptotic phenotype in fibroblasts/myofibroblasts via two important anti-apoptotic pathways: the FAK pathway and the PI3K/AKT pathway. Treatment of bleomycin-exposed mice with soluble TGFB type-II receptors (TGBR2) significantly attenuates the degree of pulmonary fibrosis (Ref. 136). Platelet-derived growth factors (PDGF) including PDGFAA and PDGFBB are critical in the survival and differentiation of mesenchymal cells during lung development, and PDGFs are also important for tissue repair following injury in adult tissues. PDGFAA (via PDGFRA) activates multiple intracellular signaling molecules that play important roles in mesenchymal cell survival, including MAP kinases and the STAT family members STAT1 and STAT3 (Ref. 137, 138). Epidermal growth factor (EGF)-treatment of fibroblasts expressing human EGF receptors blocks staurosporine-induced apoptosis by inhibiting the activation of caspase 3, in an AKT-dependent manner (Ref. 139). IL-6 enhances FAS-induced apoptosis and expression of BAX in normal cells, but inhibits apoptosis and induces expression of Bcl-2 in IPF fibroblasts indicating that dysregulated IL-6 signaling in fibroblasts from IPF may increase the resistance to fibroblasts from IPF to apoptosis (Ref. 140). Insulin-like growth factor 1 (IGF1) is a fibroblast growth and survival factor. Interstitial macrophagederived IGF1 correlates with disease severity in IPF, and the TH2 cytokines IL-4 and IL-13 stimulate macrophage expression and secretion of IGF1. Conditioned media from IL-4stimulated macrophages protects myofibroblasts from apoptosis by activation of the prosurvival kinases AKT and extracellular signal-regulated kinase (Ref. 117). Th1 and Th2 regulatory cytokines have opposite effects on fibroblast apoptosis. Pretreatment with IFN $\gamma$ or TNF- $a$ increased, whereas long-term serum-free culture and pretreatment with TGFB1 or IL-10 decreased, the apoptotic susceptibility of dermal fibroblasts in the presence of 
cycloheximide, indicating that their pro- or antifibrotic effects involve direct effects on fibroblast survival (Ref. 141).

\section{Other modulators of survival/apoptosis}

PTEN deficiency is associated with fibrosis in different organs. Inactivation of PTEN in PTEN+/+ embryonic fibroblasts increases AKT activity and suppresses apoptosis in contractile collagen matrices; inhibition of AKT activity restores the sensitivity of PTEN null cells to collagen contraction-induced apoptosis (Ref. 142, 143). Caveolin 1 (CAV1) and PTEN expression are low in myofibroblasts within IPF fibroblastic foci. The absence of CAV1 confers lung fibroblast resistance to apoptosis (Ref. 115). The PI3K/AKT signaling pathway is activated after ligation of receptor tyrosine kinases and seven transmembrane $\mathrm{G}$ protein- coupled receptors, or through integrin-associated, adhesion-mediated signaling cascades. Activated AKT may support cell survival through a number of potential mechanisms, including regulation of Bcl-2 family proteins, NF- $\mathrm{\kappa B}$, caspase 9, and forkhead family transcription factors. ET1 and TGFB1 independently promote fibroblast resistance to apoptosis through signaling pathways involving p38 MAPK and PI3K/AKT (Ref. 144).

Fibroblasts lacking THY1 are resistant to collagen gel contraction-induced apoptosis, which can be restored by re-expression of THY1 (Ref. 52). Found in inflammatory zone 1 (FIZZ1) is highly expressed in bleomycin-induced lung fibrosis. FIZZ1 is involved in pulmonary fibrosis through both induction of myofibroblast differentiation and increased or prolonged survival of myofibroblasts. FIZZ1 treatment inhibited the TNF-a-induced activities of caspase 3 and caspase 8 indicating that FIZZ1 can contribute to the pathogenesis of pulmonary fibrosis by induction of myofibroblast resistance to apoptosis (Ref. 145). Expression of TWIST1 is increased in nuclei of type II epithelial cells, macrophages, and fibroblasts in IPF lungs. Suppression of TWIST1 expression results in decreased rat lung fibroblast accumulation due to increased apoptosis, whereas TWIST1 overexpression protects the cells against several apoptotic stimuli (Ref. 146).

\section{ECM/integrin and adhesion/mechanical forces}

Myofibroblast function is critically dependent on interaction with the surrounding ECM. Receptors for the ECM, in particular the integrins, act not only to provide anchorage for fibroblasts/myofibroblasts, but also transduce signals to facilitate fibroblast decisions to survive or die. Integrin-TGFB crosstalk is important in fibrosis, cancer and wound healing (Ref. 66). Altered integrin expression stimulated by fibrotic growth factors indicates that they play an integral role in the fibrosis program (Ref. 66). Integrin-mediated TGFB1 activation is a potential therapeutic target in fibrogenic disorders (Ref. 147). Interaction of a $8 \beta 1$ integrin with FN appears to promote cell survival during serum deprivation via the PI3K pathway (Ref. 148). The Arg-Gly-Asp (RGD)-containing central cell-binding domain (CCBD) of fibronectin (FN) is required for mesenchymal cell survival and growth. FN contains three growth factor-binding domains (FN-GFBDs) that bind PDGFBB, a potent fibroblast survival and mitogenic factor. Dermal fibroblast survival requires PDGFBB stimulation in the presence of FN-GFBD, as well as adhesion to FN through the CCBD (Ref. 149). Three soluble fibronectin peptides (RGD, CS-1, and FN-C/H-V) induce apoptosis in lung fibroblasts. Treatment of fibroblasts with these peptides caused proteolysis of FAK, a tyrosine kinase involved in integrin-mediated signaling related to cell survival, suggesting 
that soluble FN peptides trigger nontransformed fibroblast apoptosis by a disruption of integrin-mediated survival signaling pathways (Ref. 150). Loss of cell adhesion or adhesion signaling can lead to a form of apoptosis called anoikis. Anoikis is likely to represent an important apoptotic mechanism in mesenchymal cells. FAK is an important mediator of integrin-dependent survival signals. Inhibition of FAK activation induces anoikis. TGFB1 protects myofibroblasts from anoikis, in part, by activation of the PI3K-AKT pathway. Thus, TGFB 1 coordinately and independently activates the FAK and AKT protein kinase pathways to confer an anoikis-resistant phenotype to myofibroblasts (Ref. 151). Integrin-linked kinase (ILK) is an intracellular protein involved in the regulation of integrin-mediated processes. ILK activity decreases in response to collagen matrix contraction, which correlates with AKT dephosphorylation and induction of fibroblast apoptosis (Ref. 152).

\section{Oxidative stress / hypoxia}

Oxidation of cysteines in proteins, such as S-glutathionylation, is known to regulate death receptor induced apoptosis (Ref. 153). HIF-1a is a major regulator of hypoxic signaling and is overexpressed in the hyperplastic epithelium of fibrotic lung. Absence of HIF-1 $a$ and increased expression of Bcl-2 in fibrotic foci support the hypothesis that dysregulated hypoxic signaling is important in fibroblast resistance to apoptosis (Ref. 154).

\section{WNT/ $\beta$-catenin signaling}

Accumulation of nuclear $\beta$-catenin, a hallmark of aberrant activated WNT signaling, has been observed in lungs of patients with IPF (Ref. 155). The WNT signaling pathway regulates apoptosis through multiple mechanisms (Ref. 156). WNT1 signaling inhibits apoptosis by activation of $\beta$-catenin/TCF-mediated transcription. WNT signaling can increase survival by activation of NF- $\mathrm{kB}$ or inhibition of GSK3B (Ref. 156). WNT5A significantly increases fibroblast proliferation, as well as relative resistance to $\mathrm{H}_{2} \mathrm{O}_{2}$-induced apoptosis in UIP lung fibroblasts (Ref. 114). IPF fibroblasts constitutively express increased basal levels of SPARC, PAI- 1 , and active $\beta$-catenin; elevated SPARC activates $\beta$-catenin, which regulates expression of PAI-1, and confers an apoptosis-resistant phenotype (Ref. 89).

\section{Myofibroblast heterogeneity with regard to survival/apoptosis}

Fibroblasts/myofibroblasts in the lung consist of phenotypically diverse subpopulations. Myofibroblasts differ in cell size, cytoskeletal arrangement, surface markers, cytokine expression profiles, and receptor expression. THY1 modulates apoptotic signaling pathways in a variety of cell types. Crosslinking antibodies to THY1 induce apoptosis in thymocytes and mesangial cells (Ref. 157, 158). Fibroblasts lacking THY1 expression are resistant to apoptosis when they contract collagen gels, even though they are more contractile. Forced expression of THY1 restores apoptotic susceptibility (Ref. 52). A recent study compared morphologic and functional characteristics of fibroblasts isolated from proximal airways and distal lung parenchyma to determine phenotypic differences (Ref. 159). These phenotypic differences may partially explain the variable responses to injury and repair between proximal airways and distal lung/parenchyma in asthma and other respiratory disease. 


\section{Section summary}

Despite the diversity of causes for fibrosis and the multiple mechanisms involved in lung fibrotic disease, a common similarity important to the disease progression is the enhanced survival of fibroblasts/myofibroblasts. Fibroblast/myofibroblast survival remains a main target for fibrotic disease, and further research toward controlling the survival of fibroblast/ myofibroblasts should eventually lead to new effective treatments. Apoptosis of fibroblasts is required for normal wound healing and limitation of collagen deposition, and resistance to apoptosis has been linked to tissue fibrosis; therefore, promoting fibroblast apoptotic signaling pathways and inhibition of anti-apoptotic pathways to limit fibroblast survival may help promote the resolution of fibrosis.

\section{Myofibroblasts as therapeutic targets (summary)}

Myofibroblast differentiation is a complex and highly regulated process. Understanding the diverse mechanisms of regulation of this phenotype offers several possible targets for intervention in fibrotic disorders and in several malignancies. An important challenge is to identify the hierarchy of pathways and discover "master switches" that control multiple pathways. TGFB1 is attractive in this regard, as it regulates most aspects of the phenotype, however it is not thought to be a good clinical target because of its many other critical biological roles. However, its activation from latent form can be selectively targeted. LSKL, a peptide antagonist of TSP-1, can attenuate renal interstitial fibrosis in rats with unilateral ureteral obstruction (Ref. 160).TGFB1 can be antagonized with blocking antibodies, or by a mutated form of TGFB3 as shown in a mouse skin wound model (Ref. 45), while the release of TGFB1 can be decreased in vivo by a lentiviral shRNA construct against CD36 (Ref. 61). Additional therapeutic candidate targets for modulating TGFB1 are PAR-1 (Ref. 60) and THY1 (Ref. 54). Other pro-myofibroblastic cytokines, chemokines and growth factors that may be useful targets include IL-4 (Ref. 43), CXCL10 (Ref. 72), SOCS1 (Ref. 161), CCR2 (Ref. 162), and CXCL12 (Ref. 29). However, many of these factors are important in early phases of fibrogenesis, soon after tissue injury. PPAR $\gamma$ agonists (e.g., troglitazone) are able to inhibit fibrosis in a murine model, even when administration is delayed until after the initial inflammatory phase has largely resolved (Ref. 82). The oxidant-antioxidant balance in in vivo lung fibrosis has been restored by NOX4 siRNA (Ref. 76) and in vitro results suggest that siRNA constructs against HIF-1a or CXCL12 can possibly be effective as well (Ref. 79). The PPAR $\gamma$-agonist troglitazone and the Wnt antagonist Dickkopf-1 reduced lung (Ref. 82) and renal fibrosis (Ref. 90) respectively. The antifibrotic drug pirfenidone, which appears to modify growth factor expression, collagen expression and redox balance, reduces myofibroblast accumulation in bleomycin-induced lung fibrosis and seems to have modest clinical benefits in IPF and renal fibrosis (Ref. 163, 164, 165, 166). The complexity of myofibroblast differentiation is demonstrated by the wide range of signaling pathways which have been suggested as therapeutic tagets. More recent candidates inlcude the Rho family of small GTPases, HH signaling, DIA, or epigenetic modifiers; targeting of which may reveal novel solutions not only for organ fibrosis but also for malignancies, pulmonary hypertension, or thyroid eye disease. 
Targeting myofibroblasts has proven an effective strategy in some models of organ fibrosis (Ref. 167); however because of remarkable fibroblast heterogeneity, targeting strategies may need to be tissue- and even disease-specific. Because there is no consensus "myofibroblastspecific" surface marker, understanding the pathways which regulate and maintain the phenotype is critical. Strategies that target single pathways may be promising in vitro but fail in vivo, particularly during the stage of progressive fibrosis. For example, the protein kinase inhibitor (PKI) imatinib mesylate inhibits platelet-derived growth factor receptor activation and fibroblast proliferation in vitro but failed during the fibrotic phase of the bleomycin model to significantly alter fibrogenic responses assessed by histopathology, collagen content, and the accumulation of myofibroblasts within the injured lung (Ref. 168). THY1 expression affects the myofibroblast phenotype broadly (expression of muscle and ECM proteins, myogenic transcription factors, contractility, resistance to apoptosis, mechanotransduction of TGFB activation (Ref. 52, 54)), and so it may be useful to promote THY1-mediated effects by better defining its mechanisms of action or regulating its expression through epigenetic therapy. In any case, targeting myofibroblast differentiation and survival will require improved understanding of the molecular networks and hierarchies involved.

New high-throughput approaches to proteomics and metabolomics could be leveraged to define non-invasive biomarkers of fibrogenesis to better monitor disease activity and response to treatment. In vitro assays for specific pathways or combinations could be used to screen compound libraries for novel antifibrotic agents. Improved understanding of the critical regulators of this complex cellular phenotype should lead to breakthroughs in the management of "classic" fibrotic diseases such as IPF and liver fibrosis, but may also offer new solutions for more common tissue remodeling disorders such as asthma and congestive heart failure, and could be useful in managing tumor invasion and metastasis regulated by myofibroblasts in tumor stroma.

\section{Acknowledgments}

Funding sources: This work was partially supported by a grant from the National Institutes of Health, National Heart Lung \& Blood Institute (HL 082818, to J.S.H.). The authors thank the peer reviewers and editors for their excellent suggestions, which have greatly strengthened the review.

\section{References}

1. Clark, RAF. Wound repair: overview and general considerations. In: Clark, RAF., editor. The Molecular and Cellular Biology of Wound Repair. Plenum Press; New York: 1996. p. 3-50.

2. Gabbiani G. The myofibroblast in wound healing and fibrocontractive diseases. J Pathol. 2003; 200:500-503. [PubMed: 12845617]

3. Hardie WD, Glasser SW, Hagood JS. Emerging concepts in the pathogenesis of lung fibrosis. Am J Pathol. 2009; 175:3-16. [PubMed: 19497999]

4. Hinz B. Formation and function of the myofibroblast during tissue repair. J Invest Dermatol. 2007; 127:526-537. [PubMed: 17299435]

5. Thiery JP, et al. Epithelial-mesenchymal transitions in development and disease. Cell. 2009; 139:871-890. [PubMed: 19945376]

6. Kalluri R, Neilson EG. Epithelial-mesenchymal transition and its implications for fibrosis. The Journal of Clinical Investigation. 2003; 112:1776-1784. [PubMed: 14679171] 
7. Zavadil J, Böttinger EP. TGF-beta and epithelial-to-mesenchymal transitions. Oncogene. 2005; 24:5764-5774. [PubMed: 16123809]

8. Iwano M, et al. Evidence that fibroblasts derive from epithelium during tissue fibrosis. The Journal of Clinical Investigation. 2002; 110:341-350. [PubMed: 12163453]

9. Osterreicher $\mathrm{CH}$, et al. Fibroblast-specific protein 1 identifies an inflammatory subpopulation of macrophages in the liver. Proc Natl Acad Sci U S A. 2011; 108:308-313. [PubMed: 21173249]

10. Zeisberg M, et al. Fibroblasts derive from hepatocytes in liver fibrosis via epithelial to mesenchymal transition. The Journal of Biological Chemistry. 2007; 282:23337-23347. [PubMed: 17562716]

11. Zeisberg EM, et al. Endothelial-to-mesenchymal transition contributes to cardiac fibrosis. Nature Medicine. 2007; 13:952-961.

12. Zeisberg EM, et al. Fibroblasts in kidney fibrosis emerge via endothelial-to-mesenchymal transition. Journal of the American Society of Nephrology: JASN. 2008; 19:2282-2287. [PubMed: 18987304]

13. Kim KK, et al. Epithelial cell alpha3beta1 integrin links beta-catenin and Smad signaling to promote myofibroblast formation and pulmonary fibrosis. The Journal of Clinical Investigation. 2009; 119:213-224. [PubMed: 19104148]

14. Câmara J, Jarai G. Epithelial-mesenchymal transition in primary human bronchial epithelial cells is Smad-dependent and enhanced by fibronectin and TNF-alpha. Fibrogenesis \& Tissue Repair. 2010; 3:2. [PubMed: 20051102]

15. Scholten D, et al. Genetic labeling does not detect epithelial-to-mesenchymal transition of cholangiocytes in liver fibrosis in mice. Gastroenterology. 2010; 139:987-998. [PubMed: 20546735]

16. Humphreys BD, et al. Fate tracing reveals the pericyte and not epithelial origin of myofibroblasts in kidney fibrosis. Am J Pathol. 2010; 176:85-97. [PubMed: 20008127]

17. Tanjore $\mathrm{H}$, et al. Contribution of epithelial-derived fibroblasts to bleomycin-induced lung fibrosis. Am J Respir Crit Care Med. 2009; 180:657-665. [PubMed: 19556518]

18. Li J, Bertram JF. Review: Endothelial-myofibroblast transition, a new player in diabetic renal fibrosis. Nephrology (Carlton, Vic). 2010; 15:507-512.

19. Nataraj D, Ernst A, Kalluri R. Idiopathic pulmonary fibrosis is associated with endothelial to mesenchymal transition. American Journal of Respiratory Cell and Molecular Biology. 2010; 43:129-130. [PubMed: 20651063]

20. Potenta S, Zeisberg E, Kalluri R. The role of endothelial-to-mesenchymal transition in cancer progression. British Journal of Cancer. 2008; 99:1375-1379. [PubMed: 18797460]

21. Widyantoro B, et al. Endothelial cell-derived endothelin-1 promotes cardiac fibrosis in diabetic hearts through stimulation of endothelial-to-mesenchymal transition. Circulation. 2010; 121:24072418. [PubMed: 20497976]

22. Hashimoto N, et al. Endothelial-mesenchymal transition in bleomycin-induced pulmonary fibrosis. American Journal of Respiratory Cell and Molecular Biology. 2010; 43:161-172. [PubMed: 19767450]

23. Arciniegas E, et al. Perspectives on endothelial-to-mesenchymal transition: potential contribution to vascular remodeling in chronic pulmonary hypertension. American Journal of Physiology Lung Cellular and Molecular Physiology. 2007; 293:L1-8. [PubMed: 17384082]

24. Hashimoto N, et al. Bone marrow-derived progenitor cells in pulmonary fibrosis. J Clin Invest. 2004; 113:243-252. [PubMed: 14722616]

25. Moeller A, et al. Circulating fibrocytes are an indicator of poor prognosis in idiopathic pulmonary fibrosis. Am J Respir Crit Care Med. 2009; 179:588-594. [PubMed: 19151190]

26. Moore BB, et al. CCR2-mediated recruitment of fibrocytes to the alveolar space after fibrotic injury. Am J Pathol. 2005; 166:675-684. [PubMed: 15743780]

27. Andersson-Sjöland A, et al. Fibrocytes are a potential source of lung fibroblasts in idiopathic pulmonary fibrosis. The International Journal of Biochemistry \& Cell Biology. 2008; 40:2129 2140. [PubMed: 18374622]

Expert Rev Mol Med. Author manuscript; available in PMC 2017 November 07. 
28. García-de-Alba C, et al. Expression of matrix metalloproteases by fibrocytes: possible role in migration and homing. American Journal of Respiratory and Critical Care Medicine. 2010; 182:1144-1152. [PubMed: 20622038]

29. Phillips RJ, et al. Circulating fibrocytes traffic to the lungs in response to CXCL12 and mediate fibrosis. J Clin Invest. 2004; 114:438-446. [PubMed: 15286810]

30. Abe R, et al. Peripheral blood fibrocytes: differentiation pathway and migration to wound sites. J Immunol. 2001; 166:7556-7562. [PubMed: 11390511]

31. Strieter RM, et al. The role of circulating mesenchymal progenitor cells, fibrocytes, in promoting pulmonary fibrosis. Transactions of the American Clinical and Climatological Association. 2009; 120:49-59. [PubMed: 19768162]

32. Asanuma H, et al. Arterially delivered mesenchymal stem cells prevent obstruction-induced renal fibrosis. J Surg Res. 2011; 168:e51-59. [PubMed: 20850784]

33. Popova AP, et al. Autocrine production of TGF-beta1 promotes myofibroblastic differentiation of neonatal lung mesenchymal stem cells. American Journal of Physiology Lung Cellular and Molecular Physiology. 2010; 298:L735-743. [PubMed: 20190033]

34. Lee CH, et al. CTGF directs fibroblast differentiation from human mesenchymal stem/stromal cells and defines connective tissue healing in a rodent injury model. The Journal of Clinical Investigation. 2010; 120:3340-3349. [PubMed: 20679726]

35. Atamas SP, White B. Cytokine regulation of pulmonary fibrosis in scleroderma. Cytokine Growth Factor Rev. 2003; 14:537-550. [PubMed: 14563355]

36. Knight DA, et al. The role of gp130/IL-6 cytokines in the development of pulmonary fibrosis: critical determinants of disease susceptibility and progression? Pharmacol Ther. 2003; 99:327338. [PubMed: 12951164]

37. Lasky JA, Brody AR. Interstitial fibrosis and growth factors. Environmental Health Perspectives. 2000; 108(Suppl 4):751-762. [PubMed: 10931794]

38. Yang L, et al. IL-21 and TGF-beta are required for differentiation of human T(H)17 cells. Nature. 2008; 454:350-352. [PubMed: 18469800]

39. Wilson MS, et al. Bleomycin and IL-1beta-mediated pulmonary fibrosis is IL-17A dependent. J Exp Med. 2010; 207:535-552. [PubMed: 20176803]

40. Yagi Y, et al. Inflammatory responses induced by interleukin-17 family members in human colonic subepithelial myofibroblasts. Journal of Gastroenterology. 2007; 42:746-753. [PubMed: 17876544]

41. Turner NA, et al. Interleukin-1alpha stimulates proinflammatory cytokine expression in human cardiac myofibroblasts. American Journal of Physiology Heart and Circulatory Physiology. 2009; 297:H1117-1127. [PubMed: 19648252]

42. Collard HR, et al. Plasma biomarker profiles in acute exacerbation of idiopathic pulmonary fibrosis. American Journal of Physiology Lung Cellular and Molecular Physiology. 2010; 299:L37. [PubMed: 20418386]

43. Huaux F, et al. Dual roles of IL-4 in lung injury and fibrosis. Journal of Immunology (Baltimore, Md: 1950). 2003; 170:2083-2092.

44. Zhou Y, Hagood JS, Murphy-Ullrich JE. Thy-1 Expression Regulates the Ability of Rat Lung Fibroblasts to Activate Transforming Growth Factor- $\{$ beta $\}$ in Response to Fibrogenic Stimuli. Am J Pathol. 2004; 165:659-669. [PubMed: 15277239]

45. Waddington SN, et al. Gene Delivery of a Mutant TGF 33 Reduces Markers of Scar Tissue Formation After Cutaneous Wounding. Molecular Therapy: The Journal of the American Society of Gene Therapy. 2010; 18:2104-2111. [PubMed: 20736928]

46. Liu X, Kelm RJ, Strauch AR. Transforming growth factor beta1-mediated activation of the smooth muscle alpha-actin gene in human pulmonary myofibroblasts is inhibited by tumor necrosis factoralpha via mitogen-activated protein kinase kinase 1-dependent induction of the Egr-1 transcriptional repressor. Molecular Biology of the Cell. 2009; 20:2174-2185. [PubMed: 19261809]

47. Bhatnagar $\mathrm{S}$, et al. Transforming growth factor-beta-activated kinase 1 is an essential regulator of myogenic differentiation. The Journal of Biological Chemistry. 2010; 285:6401-6411. [PubMed: 20037161] 
48. Liu T, et al. FIZZ1 stimulation of myofibroblast differentiation. Am J Pathol. 2004; 164:13151326. [PubMed: 15039219]

49. Lin X, et al. Yin Yang 1 is a novel regulator of pulmonary fibrosis. Am J Respir Crit Care Med. 2010

50. Hagood JS, et al. Loss of fibroblast Thy-1 expression correlates with lung fibrogenesis. Am J Pathol. 2005; 167:365-379. [PubMed: 16049324]

51. Sanders YY, et al. Epigenetic regulation mediates thy-1 gene silencing and induction of the myofibroblast phenotype. Proc Am Thorac Soc. 2008; 5:363-364.

52. Sanders YY, Kumbla P, Hagood JS. Enhanced myofibroblastic differentiation and survival in Thy-1(-) lung fibroblasts. Am J Respir Cell Mol Biol. 2007; 36:226-235. [PubMed: 16960126]

53. Sanders YY, et al. Thy-1 Promoter Hypermethylation: A Novel Epigenetic Pathogenic Mechanism in Pulmonary Fibrosis. Am J Respir Cell Mol Biol. 2008; 39:610-618. [PubMed: 18556592]

54. Zhou Y, et al. Thy-1-integrin alphav beta5 interactions inhibit lung fibroblast contraction-induced latent transforming growth factor-beta1 activation and myofibroblast differentiation. J Biol Chem. 2010; 285:22382-22393. [PubMed: 20463011]

55. Koumas L, et al. Thy-1 expression in human fibroblast subsets defines myofibroblastic or lipofibroblastic phenotypes. Am J Pathol. 2003; 163:1291-1300. [PubMed: 14507638]

56. Lehmann GM, et al. Novel anti-adipogenic activity produced by human fibroblasts. Am J Physiol Cell Physiol. 2010; 299:C672-681. [PubMed: 20554910]

57. Eckes B, et al. Mechanical tension and integrin alpha 2 beta 1 regulate fibroblast functions. The Journal of Investigative Dermatology. Symposium Proceedings / the Society for Investigative Dermatology, Inc. [and] European Society for Dermatological Research. 2006; 11:66-72.

58. Tomasek JJ, et al. Myofibroblasts and mechano-regulation of connective tissue remodelling. Nat Rev Mol Cell Biol. 2002; 3:349-363. [PubMed: 11988769]

59. Katsumi A, et al. Integrins in mechanotransduction. The Journal of Biological Chemistry. 2004; 279:12001-12004. [PubMed: 14960578]

60. Jenkins RG, et al. Ligation of protease-activated receptor 1 enhances alpha(v)beta6 integrindependent TGF-beta activation and promotes acute lung injury. The Journal of Clinical Investigation. 2006; 116:1606-1614. [PubMed: 16710477]

61. Wang X, et al. Silencing CD36 gene expression results in the inhibition of latent-TGF-beta1 activation and suppression of silica-induced lung fibrosis in the rat. Respiratory Research. 2009; 10:36. [PubMed: 19439069]

62. Wipff PJ, et al. Myofibroblast contraction activates latent TGF-beta1 from the extracellular matrix. The Journal of Cell Biology. 2007; 179:1311-1323. [PubMed: 18086923]

63. Zhou Y, et al. Latent transforming growth factor-beta-binding protein-4 regulates transforming growth factor-beta1 bioavailability for activation by fibrogenic lung fibroblasts in response to bleomycin. Am J Pathol. 2009; 174:21-33. [PubMed: 19056849]

64. Giancotti FG, Ruoslahti E. Integrin signaling. Science. 1999; 285:1028-1032. [PubMed: 10446041]

65. Choi SS, et al. Activation of Rac1 promotes hedgehog-mediated acquisition of the myofibroblastic phenotype in rat and human hepatic stellate cells. Hepatology (Baltimore, Md). 2010; 52:278-290.

66. Margadant C, Sonnenberg A. Integrin-TGF-beta crosstalk in fibrosis, cancer and wound healing. EMBO Reports. 2010; 11:97-105. [PubMed: 20075988]

67. Chan MWC, et al. Force-induced myofibroblast differentiation through collagen receptors is dependent on mammalian diaphanous (mDia). The Journal of Biological Chemistry. 2010; 285:9273-9281. [PubMed: 20071339]

68. Follonier Castella L, et al. Regulation of myofibroblast activities: calcium pulls some strings behind the scene. Experimental Cell Research. 2010; 316:2390-2401. [PubMed: 20451515]

69. Couchman JR, Woods A. Syndecans, signaling, and cell adhesion. Journal of Cellular Biochemistry. 1996; 61:578-584. [PubMed: 8806081]

70. Yoneda A, Couchman JR. Regulation of cytoskeletal organization by syndecan transmembrane proteoglycans. Matrix Biology: Journal of the International Society for Matrix Biology. 2003; 22:25-33. [PubMed: 12714039] 
71. Kennedy L, et al. CCN2 is necessary for the function of mouse embryonic fibroblasts. Experimental Cell Research. 2007; 313:952-964. [PubMed: 17239853]

72. Jiang D, et al. Inhibition of pulmonary fibrosis in mice by CXCL10 requires glycosaminoglycan binding and syndecan-4. J Clin Invest. 2010; 120:2049-2057. [PubMed: 20484822]

73. Avalos AM, et al. Neuronal Thy-1 induces astrocyte adhesion by engaging syndecan- 4 in a cooperative interaction with alphavbeta3 integrin that activates PKCalpha and RhoA. J Cell Sci. 2009; 122:3462-3471. [PubMed: 19723805]

74. Leto TL, et al. Targeting and regulation of reactive oxygen species generation by Nox family NADPH oxidases. Antioxidants \& Redox Signaling. 2009; 11:2607-2619. [PubMed: 19438290]

75. Kinnula VL, et al. Oxidative stress in pulmonary fibrosis: a possible role for redox modulatory therapy. American Journal of Respiratory and Critical Care Medicine. 2005; 172:417-422. [PubMed: 15894605]

76. Hecker L, et al. NADPH oxidase-4 mediates myofibroblast activation and fibrogenic responses to lung injury. Nat Med. 2009; 15:1077-1081. [PubMed: 19701206]

77. Cucoranu I, et al. NAD(P)H oxidase 4 mediates transforming growth factor-beta1-induced differentiation of cardiac fibroblasts into myofibroblasts. Circulation Research. 2005; 97:900-907. [PubMed: 16179589]

78. Amara N, et al. NOX4/NADPH oxidase expression is increased in pulmonary fibroblasts from patients with idiopathic pulmonary fibrosis and mediates TGFbeta1-induced fibroblast differentiation into myofibroblasts. Thorax. 2010; 65:733-738. [PubMed: 20685750]

79. Toullec A, et al. Oxidative stress promotes myofibroblast differentiation and tumour spreading. EMBO Molecular Medicine. 2010; 2:211-230. [PubMed: 20535745]

80. Van Rheen Z, et al. Lung extracellular superoxide dismutase overexpression lessens bleomycininduced pulmonary hypertension and vascular remodeling. Am J Respir Cell Mol Biol. 2011; 44:500-508. [PubMed: 20539010]

81. Burgess HA, et al. PPARgamma agonists inhibit TGF-beta induced pulmonary myofibroblast differentiation and collagen production: implications for therapy of lung fibrosis. Am J Physiol Lung Cell Mol Physiol. 2005; 288:L1146-1153. [PubMed: 15734787]

82. Milam JE, et al. PPAR-gamma agonists inhibit profibrotic phenotypes in human lung fibroblasts and bleomycin-induced pulmonary fibrosis. American Journal of Physiology Lung Cellular and Molecular Physiology. 2008; 294:L891-901. [PubMed: 18162602]

83. Lin Q, et al. Rosiglitazone inhibits migration, proliferation, and phenotypic differentiation in cultured human lung fibroblasts. Experimental Lung Research. 2010; 36:120-128. [PubMed: 20205597]

84. Kulkarni AA, et al. PPAR- $\gamma$ Ligands Repress TGF $\beta$-Induced Myofibroblast Differentiation by Targeting the PI3K/Akt Pathway: Implications for Therapy of Fibrosis. PLoS One. 2011; 6:e15909. [PubMed: 21253589]

85. Ferguson HE, et al. Electrophilic peroxisome proliferator-activated receptor-gamma ligands have potent antifibrotic effects in human lung fibroblasts. American Journal of Respiratory Cell and Molecular Biology. 2009; 41:722-730. [PubMed: 19286977]

86. Ferguson HE, et al. Peroxisome proliferator-activated receptor-gamma ligands induce heme oxygenase-1 in lung fibroblasts by a PPARgamma-independent, glutathione-dependent mechanism. American Journal of Physiology Lung Cellular and Molecular Physiology. 2009; 297:L912-919. [PubMed: 19734319]

87. Fagone E, et al. Resveratrol inhibits transforming growth factor-beta-induced proliferation and differentiation of ex vivo human lung fibroblasts into myofibroblasts through ERK/Akt inhibition and PTEN restoration. Exp Lung Res. 2011; 37:162-174. [PubMed: 21269063]

88. Kikuchi A, Yamamoto H, Kishida S. Multiplicity of the interactions of Wnt proteins and their receptors. Cellular Signalling. 2007; 19:659-671. [PubMed: 17188462]

89. Chang W, et al. SPARC suppresses apoptosis of idiopathic pulmonary fibrosis fibroblasts through constitutive activation of beta-catenin. The Journal of Biological Chemistry. 2010; 285:8196-8206. [PubMed: 20061390]

90. He W, et al. Wnt/beta-catenin signaling promotes renal interstitial fibrosis. Journal of the American Society of Nephrology: JASN. 2009; 20:765-776. [PubMed: 19297557] 
91. Lee SH, et al. ERK activation drives intestinal tumorigenesis in $\mathrm{Apc}(\mathrm{min} /+)$ mice. Nature Medicine. 2010; 16:665-670.

92. Egger G, et al. Epigenetics in human disease and prospects for epigenetic therapy. Nature. 2004; 429:457-463. [PubMed: 15164071]

93. Reik W. Stability and flexibility of epigenetic gene regulation in mammalian development. Nature. 2007; 447:425-432. [PubMed: 17522676]

94. Laurent L, et al. Dynamic changes in the human methylome during differentiation. Genome Res. 2010; 20:320-331. [PubMed: 20133333]

95. Wang Y, Fan PS, Kahaleh B. Association between enhanced type I collagen expression and epigenetic repression of the FLI1 gene in scleroderma fibroblasts. Arthritis Rheum. 2006; 54:2271-2279. [PubMed: 16802366]

96. Glenisson W, Castronovo V, Waltregny D. Histone deacetylase 4 is required for TGFbeta1-induced myofibroblastic differentiation. Biochim Biophys Acta. 2007; 1773:1572-1582. [PubMed: 17610967]

97. Guo W, et al. Abrogation of TGF-beta1-induced fibroblast-myofibroblast differentiation by histone deacetylase inhibition. Am J Physiol Lung Cell Mol Physiol. 2009; 297:L864-870. [PubMed: 19700647]

98. Hu B, et al. Epigenetic regulation of myofibroblast differentiation by DNA methylation. Am J Pathol. 2010; 177:21-28. [PubMed: 20489138]

99. van Rooij E, et al. Dysregulation of microRNAs after myocardial infarction reveals a role of miR-29 in cardiac fibrosis. Proc Natl Acad Sci U S A. 2008; 105:13027-13032. [PubMed: 18723672]

100. Liu G, et al. miR-21 mediates fibrogenic activation of pulmonary fibroblasts and lung fibrosis. J Exp Med. 2010; 207:1589-1597. [PubMed: 20643828]

101. Yao Q, et al. Micro-RNA-21 regulates TGF-beta-induced myofibroblast differentiation by targeting PDCD4 in tumor-stroma interaction. Int J Cancer. 2011; 128:1783-1792. [PubMed: 20533548]

102. Mann J, et al. MeCP2 controls an epigenetic pathway that promotes myofibroblast transdifferentiation and fibrosis. Gastroenterology. 2010; 138:705-714. 714 e701-704. [PubMed: 19843474]

103. Chabaud S, et al. Decreased secretion of MMP by non-lesional late-stage scleroderma fibroblasts after selection via activation of the apoptotic fas-pathway. J Cell Physiol. 2011; 226:1907-1914. [PubMed: 21506121]

104. Fattman CL. Apoptosis in pulmonary fibrosis: too much or not enough? Antioxid Redox Signal. 2008; 10:379-385. [PubMed: 18031201]

105. Maher TM, et al. Diminished prostaglandin E2 contributes to the apoptosis paradox in idiopathic pulmonary fibrosis. Am J Respir Crit Care Med. 2010; 182:73-82. [PubMed: 20203246]

106. Jinta $\mathrm{T}$, et al. The pathogenesis of chronic hypersensitivity pneumonitis in common with idiopathic pulmonary fibrosis: expression of apoptotic markers. Am J Clin Pathol. 2010; 134:613-620. [PubMed: 20855643]

107. Kuhn C, McDonald JA. The roles of the myofibroblast in idiopathic pulmonary fibrosis. Ultrastructural and immunohistochemical features of sites of active extracellular matrix synthesis. Am J Pathol. 1991; 138:1257-1265. [PubMed: 2024710]

108. Drakopanagiotakis F, et al. Apoptosis in lung injury and fibrosis. Eur Respir J. 2008; 32:16311638. [PubMed: 19043009]

109. Beon M, et al. Myofibroblast induction and microvascular alteration in scleroderma lung fibrosis. Clin Exp Rheumatol. 2004; 22:733-742. [PubMed: 15638048]

110. Uhal BD. Apoptosis in lung fibrosis and repair. Chest. 2002; 122:293S-298S. [PubMed: 12475803]

111. Vittal R, et al. Modulation of prosurvival signaling in fibroblasts by a protein kinase inhibitor protects against fibrotic tissue injury. Am J Pathol. 2005; 166:367-375. [PubMed: 15681821]

112. Horowitz JC, et al. Activation of the pro-survival phosphatidylinositol 3-kinase/AKT pathway by transforming growth factor-beta1 in mesenchymal cells is mediated by p38 MAPK-dependent

Expert Rev Mol Med. Author manuscript; available in PMC 2017 November 07. 
induction of an autocrine growth factor. J Biol Chem. 2004; 279:1359-1367. [PubMed: 14576166]

113. Garneau-Tsodikova S, Thannickal VJ. Protein kinase inhibitors in the treatment of pulmonary fibrosis. Curr Med Chem. 2008; 15:2632-2640. [PubMed: 18855683]

114. Vuga LJ, et al. WNT5A is a regulator of fibroblast proliferation and resistance to apoptosis. Am J Respir Cell Mol Biol. 2009; 41:583-589. [PubMed: 19251946]

115. Xia H, et al. Pathologic caveolin-1 regulation of PTEN in idiopathic pulmonary fibrosis. The American Journal of Pathology. 2010; 176:2626-2637. [PubMed: 20395445]

116. Huang SK, et al. Prostaglandin E(2) induces fibroblast apoptosis by modulating multiple survival pathways. FASEB J. 2009; 23:4317-4326. [PubMed: 19671668]

117. Wynes MW, Frankel SK, Riches DW. IL-4-induced macrophage-derived IGF-I protects myofibroblasts from apoptosis following growth factor withdrawal. J Leukoc Biol. 2004; 76:1019-1027. [PubMed: 15316031]

118. Selman M, Pardo A. Idiopathic pulmonary fibrosis: misunderstandings between epithelial cells and fibroblasts? Sarcoidosis Vasc Diffuse Lung Dis. 2004; 21:165-172. [PubMed: 15554072]

119. Chapman HA. Epithelial-mesenchymal interactions in pulmonary fibrosis. Annu Rev Physiol. 2011; 73:413-435. [PubMed: 21054168]

120. Horowitz JC, Thannickal VJ. Epithelial-mesenchymal interactions in pulmonary fibrosis. Semin Respir Crit Care Med. 2006; 27:600-612. [PubMed: 17195137]

121. Selman M, Pardo A. Role of epithelial cells in idiopathic pulmonary fibrosis: from innocent targets to serial killers. Proc Am Thorac Soc. 2006; 3:364-372. [PubMed: 16738202]

122. Golan-Gerstl R, et al. Epithelial cell apoptosis by fas ligand-positive myofibroblasts in lung fibrosis. Am J Respir Cell Mol Biol. 2007; 36:270-275. [PubMed: 16990614]

123. Kuwano K, et al. Essential roles of the Fas-Fas ligand pathway in the development of pulmonary fibrosis. J Clin Invest. 1999; 104:13-19. [PubMed: 10393694]

124. Aoshiba K, et al. The Fas/Fas-ligand system is not required for bleomycin-induced pulmonary fibrosis in mice. Am J Respir Crit Care Med. 2000; 162:695-700. [PubMed: 10934108]

125. Moodley YP, et al. Comparison of the morphological and biochemical changes in normal human lung fibroblasts and fibroblasts derived from lungs of patients with idiopathic pulmonary fibrosis during FasL-induced apoptosis. J Pathol. 2004; 202:486-495. [PubMed: 15095276]

126. Buhling F, et al. Altered expression of membrane-bound and soluble CD95/Fas contributes to the resistance of fibrotic lung fibroblasts to FasL induced apoptosis. Respir Res. 2005; 6:37. [PubMed: 15833141]

127. Kolodsick JE, et al. Prostaglandin E2 inhibits fibroblast to myofibroblast transition via E. prostanoid receptor 2 signaling and cyclic adenosine monophosphate elevation. Am J Respir Cell Mol Biol. 2003; 29:537-544. [PubMed: 12738687]

128. Wilborn J, et al. Cultured lung fibroblasts isolated from patients with idiopathic pulmonary fibrosis have a diminished capacity to synthesize prostaglandin E2 and to express cyclooxygenase-2. J Clin Invest. 1995; 95:1861-1868. [PubMed: 7706493]

129. Horowitz JC, Peters-Golden M. Prostaglandin E2's new trick: "decider" of differential alveolar cell life and death. Am J Respir Crit Care Med. 2010; 182:2-3. [PubMed: 20601589]

130. Hagimoto N, et al. TGF-beta 1 as an enhancer of Fas-mediated apoptosis of lung epithelial cells. J Immunol. 2002; 168:6470-6478. [PubMed: 12055267]

131. Chodon T, et al. Keloid-derived fibroblasts are refractory to Fas-mediated apoptosis and neutralization of autocrine transforming growth factor-betal can abrogate this resistance. Am J Pathol. 2000; 157:1661-1669. [PubMed: 11073825]

132. Tanaka T, et al. Resistance to Fas-mediated apoptosis in human lung fibroblast. Eur Respir J. 2002; 20:359-368. [PubMed: 12212968]

133. Kazufumi M, et al. Expression of bcl-2 protein and APO-1 (Fas antigen) in the lung tissue from patients with idiopathic pulmonary fibrosis. Microsc Res Tech. 1997; 38:480-487. [PubMed: 9376651]

134. Marcotte R, Lacelle C, Wang E. Senescent fibroblasts resist apoptosis by downregulating caspase-3. Mech Ageing Dev. 2004; 125:777-783. [PubMed: 15541772] 
135. Budinger GR, et al. Proapoptotic Bid is required for pulmonary fibrosis. Proc Natl Acad Sci U S A. 2006; 103:4604-4609. [PubMed: 16537427]

136. Yamada M, et al. Gene transfer of soluble transforming growth factor type II receptor by in vivo electroporation attenuates lung injury and fibrosis. J Clin Pathol. 2007; 60:916-920. [PubMed: 17018685]

137. Bonner JC. Regulation of PDGF and its receptors in fibrotic diseases. Cytokine Growth Factor Rev. 2004; 15:255-273. [PubMed: 15207816]

138. Bonner JC. Mesenchymal cell survival in airway and interstitial pulmonary fibrosis. Fibrogenesis Tissue Repair. 2010; 3:15. [PubMed: 20738867]

139. Shao H, Yi XM, Wells A. Epidermal growth factor protects fibroblasts from apoptosis via PI3 kinase and Rac signaling pathways. Wound Repair Regen. 2008; 16:551-558. [PubMed: 18638274]

140. Moodley YP, et al. Inverse effects of interleukin-6 on apoptosis of fibroblasts from pulmonary fibrosis and normal lungs. Am J Respir Cell Mol Biol. 2003; 29:490-498. [PubMed: 12714376]

141. Santiago B, et al. Intracellular regulation of Fas-induced apoptosis in human fibroblasts by extracellular factors and cycloheximide. J Immunol. 2004; 172:560-566. [PubMed: 14688367]

142. White ES, et al. Negative regulation of myofibroblast differentiation by PTEN (Phosphatase and Tensin Homolog Deleted on chromosome 10). Am J Respir Crit Care Med. 2006; 173:112-121. [PubMed: 16179636]

143. Nho RS, et al. PTEN regulates fibroblast elimination during collagen matrix contraction. J Biol Chem. 2006; 281:33291-33301. [PubMed: 16963781]

144. Kulasekaran P, et al. Endothelin-1 and transforming growth factor-beta1 independently induce fibroblast resistance to apoptosis via AKT activation. Am J Respir Cell Mol Biol. 2009; 41:484493. [PubMed: 19188658]

145. Chung MJ, et al. Antiapoptotic effect of found in inflammatory zone (FIZZ)1 on mouse lung fibroblasts. J Pathol. 2007; 212:180-187. [PubMed: 17492827]

146. Bridges RS, et al. Gene expression profiling of pulmonary fibrosis identifies Twist 1 as an antiapoptotic molecular "rectifier" of growth factor signaling. Am J Pathol. 2009; 175:23512361. [PubMed: 19893041]

147. Nishimura SL. Integrin-mediated transforming growth factor-beta activation, a potential therapeutic target in fibrogenic disorders. Am J Pathol. 2009; 175:1362-1370. [PubMed: 19729474]

148. Farias E, et al. Integrin alpha8beta1-fibronectin interactions promote cell survival via PI3 kinase pathway. Biochem Biophys Res Commun. 2005; 329:305-311. [PubMed: 15721307]

149. Lin F, et al. Fibronectin growth factor-binding domains are required for fibroblast survival. J Invest Dermatol. 2011; 131:84-98. [PubMed: 20811396]

150. Hadden HL, Henke CA. Induction of lung fibroblast apoptosis by soluble fibronectin peptides. Am J Respir Crit Care Med. 2000; 162:1553-1560. [PubMed: 11029376]

151. Horowitz JC, et al. Combinatorial activation of FAK and AKT by transforming growth factorbeta1 confers an anoikis-resistant phenotype to myofibroblasts. Cell Signal. 2007; 19:761-771. [PubMed: 17113264]

152. Nho RS, et al. Role of integrin-linked kinase in regulating phosphorylation of Akt and fibroblast survival in type I collagen matrices through a beta1 integrin viability signaling pathway. J Biol Chem. 2005; 280:26630-26639. [PubMed: 15905178]

153. Janssen-Heininger YM, et al. Regulation of apoptosis through cysteine oxidation: implications for fibrotic lung disease. Ann N Y Acad Sci. 2010; 1203:23-28. [PubMed: 20716279]

154. Tzouvelekis A, et al. Comparative expression profiling in pulmonary fibrosis suggests a role of hypoxia-inducible factor-1alpha in disease pathogenesis. Am J Respir Crit Care Med. 2007; 176:1108-1119. [PubMed: 17761615]

155. Chilosi M, et al. Aberrant Wnt/beta-catenin pathway activation in idiopathic pulmonary fibrosis. Am J Pathol. 2003; 162:1495-1502. [PubMed: 12707032]

156. Pecina-Slaus N. Wnt signal transduction pathway and apoptosis: a review. Cancer Cell Int. 2010; 10:22. [PubMed: 20591184] 
157. Hueber AO, et al. Thy-1 triggers mouse thymocyte apoptosis through a bcl-2-resistant mechanism. J Exp Med. 1994; 179:785-796. [PubMed: 7906706]

158. Morita $\mathrm{H}$, et al. Thy-1 antigen mediates apoptosis of rat glomerular cells in vitro and in vivo. Nephron. 1996; 73:293-298. [PubMed: 8773358]

159. Kotaru C, et al. Regional fibroblast heterogeneity in the lung: implications for remodeling. Am J Respir Crit Care Med. 2006; 173:1208-1215. [PubMed: 16543551]

160. Xie, X-s, et al. LSKL, a peptide antagonist of thrombospondin-1, attenuates renal interstitial fibrosis in rats with unilateral ureteral obstruction. Archives of Pharmacal Research. 2010; 33:275-284. [PubMed: 20195829]

161. Nakashima T, et al. Suppressor of cytokine signaling 1 inhibits pulmonary inflammation and fibrosis. The Journal of Allergy and Clinical Immunology. 2008; 121:1269-1276. [PubMed: 18355908]

162. Moore BB, et al. Protection from pulmonary fibrosis in the absence of CCR2 signaling. J Immunol. 2001; 167:4368-4377. [PubMed: 11591761]

163. Cho ME, et al. Pirfenidone slows renal function decline in patients with focal segmental glomerulosclerosis. Clin J Am Soc Nephrol. 2007; 2:906-913. [PubMed: 17702727]

164. Kakugawa T, et al. Pirfenidone attenuates expression of HSP47 in murine bleomycin-induced pulmonary fibrosis. Eur Respir J. 2004; 24:57-65. [PubMed: 15293605]

165. Lasky J. Pirfenidone. IDrugs. 2004; 7:166-172. [PubMed: 15057662]

166. Noble PW, et al. Pirfenidone in patients with idiopathic pulmonary fibrosis (CAPACITY): two randomised trials. Lancet. 2011; 377:1760-1769. [PubMed: 21571362]

167. Douglass A, et al. Antibody-targeted myofibroblast apoptosis reduces fibrosis during sustained liver injury. J Hepatol. 2008; 49:88-98. [PubMed: 18394744]

168. Vittal R, et al. Effects of the protein kinase inhibitor, imatinib mesylate, on epithelial/ mesenchymal phenotypes: implications for treatment of fibrotic diseases. J Pharmacol Exp Ther. 2007; 321:35-44. [PubMed: 17218487]

169. Kisseleva T, Brenner DA. Is it the end of the line for the EMT? Hepatology. 2011; 53:1433-1435. [PubMed: 21433040]

170. Kinoshita K, et al. Adenovirus-mediated expression of BMP-7 suppresses the development of liver fibrosis in rats. Gut. 2007; 56:706-714. [PubMed: 17127702]

171. Crosby LM, Waters CM. Epithelial repair mechanisms in the lung. American Journal of Physiology Lung Cellular and Molecular Physiology. 2010; 298:L715-731. [PubMed: 20363851]

172. Nho R, Kahm J. $\beta 1$-Integrin-Collagen Interaction Suppresses FoxO3a by the Coordination of Akt and PP2A. J Biol Chem. 2010; 285:14195-14209. [PubMed: 20223831]

173. Wang XM, et al. Caveolin-1: a critical regulator of lung fibrosis in idiopathic pulmonary fibrosis. J Exp Med. 2006; 203:2895-2906. [PubMed: 17178917]

\section{Further reading}

In addition to review articles cited above, the reader is directed to the following articles and websites of interest:

174. Hinz B. Formation and function of the myofibroblast during tissue repair. J Invest Dermatol. 2007; 127:526-537. An excellent and comprehensive review from a leader in the field. [PubMed: 17299435]

175. Hinz B. The myofibroblast: paradigm for a mechanically active cell. J Biomech. 2010; 43:146155. A concise review of the fundamental character of the myofibroblast and the known biomechanics of myofibroblast differentiation. [PubMed: 19800625]

176. Wipff PJ, et al. Myofibroblast contraction activates latent TGF-beta1 from the extracellular matrix. The Journal of Cell Biology. 2007; 179:1311-1323. An important study in the field demonstrating a mechanism for mechanical force-mediated activation of myofibroblasts. [PubMed: 18086923] 
177. Kisseleva T, et al. Is it the end of the line for the EMT? Hepatology. 2011; 53:1433-1435. (Ref. 169). An interesting recent editorial outlining the controversy regarding EMT in the liver. [PubMed: 21433040]

178. http://www.cellmigration.org/ A well-maintained websitewith excellent figures and movies, brief reviews, and helpful external links

179. http://www.asmb.net/ Website of the American Society for Matrix Biology, with meeting announcements, resources, and external links 


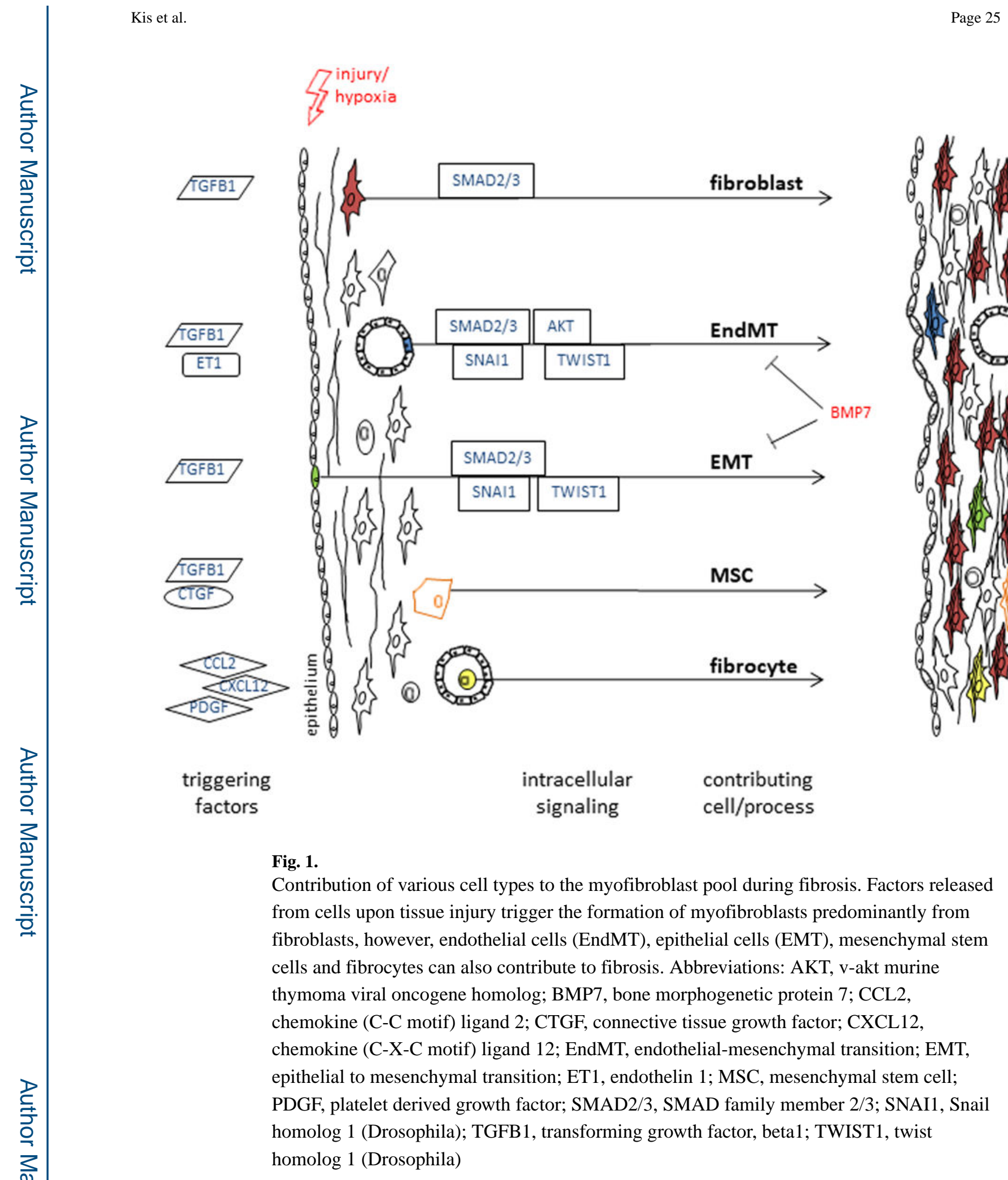

Expert Rev Mol Med. Author manuscript; available in PMC 2017 November 07. 


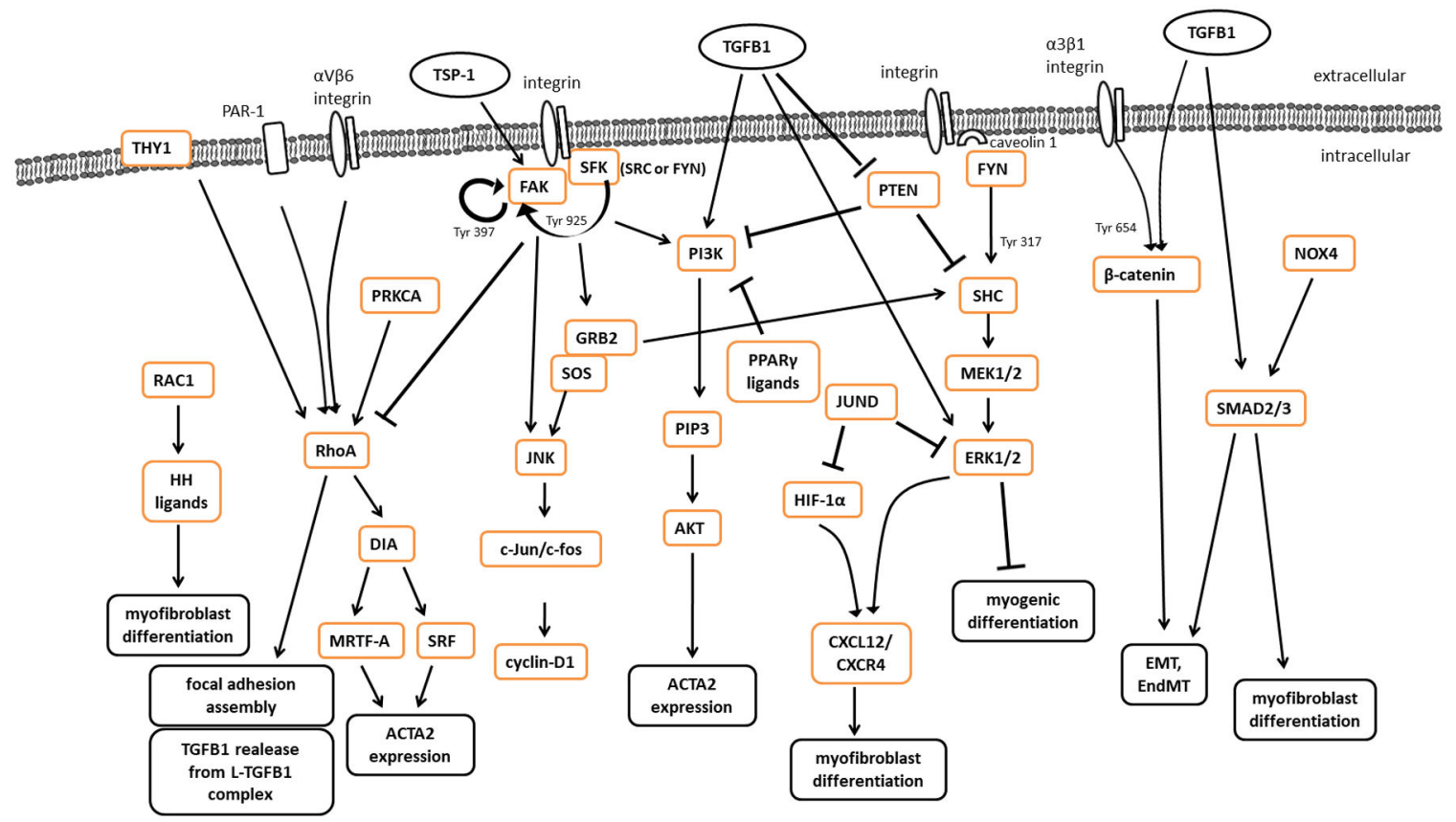

Fig. 2.

Network of selected factors influencing myofibroblast differentiation. Several integrinactivated signaling pathways (e.g. FAK/JNK/AP-1, PI3K/AKT or MEK1/2/ERK1/2) are involved in myofibroblast differentiation. The potent profibrotic effect of TGFB1 is demonstrated by its influence on multiple pathways. Recent results showing the role of $\mathrm{HH}$ ligands, DIA, PPAR $\gamma$ ligands, HIF-1a and NOX4 are also illustrated (see text of article; the figure is not comprehensive). Abbreviations: ACTA2, actin, alpha 2, smooth muscle; AKT, $\mathrm{v}$-akt murine thymoma viral oncogene homolog; c-fos, FBJ murine osteosarcoma viral oncogene homolog ; c-Jun, jun proto-oncogene; CTGF, connective tissue growth factor; CXCL12, chemokine (C-X-C motif) ligand 12; CXCR4, chemokine (C-X-C motif) receptor 4 ; DIA, diaphanous homolog 1 (Drosophila); EMT, epithelial to mesenchymal transition; EndMT, endothelial-mesenchymal transition; ERK1/2, mitogen-activated protein kinase 1/2; FAK, PTK2 protein tyrosine kinase 2; FYN, FYN oncogene related to SRC, FGR, YES; GRB2, growth factor receptor-bound protein 2; HH ligand, Hedgehog ligand; HIF-1a, hypoxia inducible factor 1, alpha subunit; JNK, JUN N-terminal kinase; JUND, jun D protooncogene; L-TGFB1, latent transforming growth factor, beta1; MEK1/2, mitogen-activated protein kinase kinase 1/2; MRTF-A, myocardin-related transcription factor A; NOX4, NADPH oxidase 4; PAR-1, coagulation factor II (thrombin) receptor; PI3K, phosphoinositide-3-kinase; PIP3, phosphatidylinositol 3,4,5-triphosphate; PRKCA, protein kinase $\mathrm{C}$, alpha; PPAR $\gamma$, peroxisome proliferator-activated receptor gamma; PTEN, phosphatase and tensin homolog; RAC1, ras-related C3 botulinum toxin substrate 1; RhoA, ras homolog gene family, member A; SMAD2/3, SMAD family member 2/3; SRC, v-src sarcoma viral oncogene homolog; SFK, SRC family kinase; SHC, SHC (Src homology 2 domain containing) transforming protein; SOS, son of sevenless homolog (Drosophila); SRF, serum response factor; TGFB1, transforming growth factor, beta1; THY1, Thy-1 cell surface antigen; TSP-1, thrombospondin 1; Tyr, tyrosine 


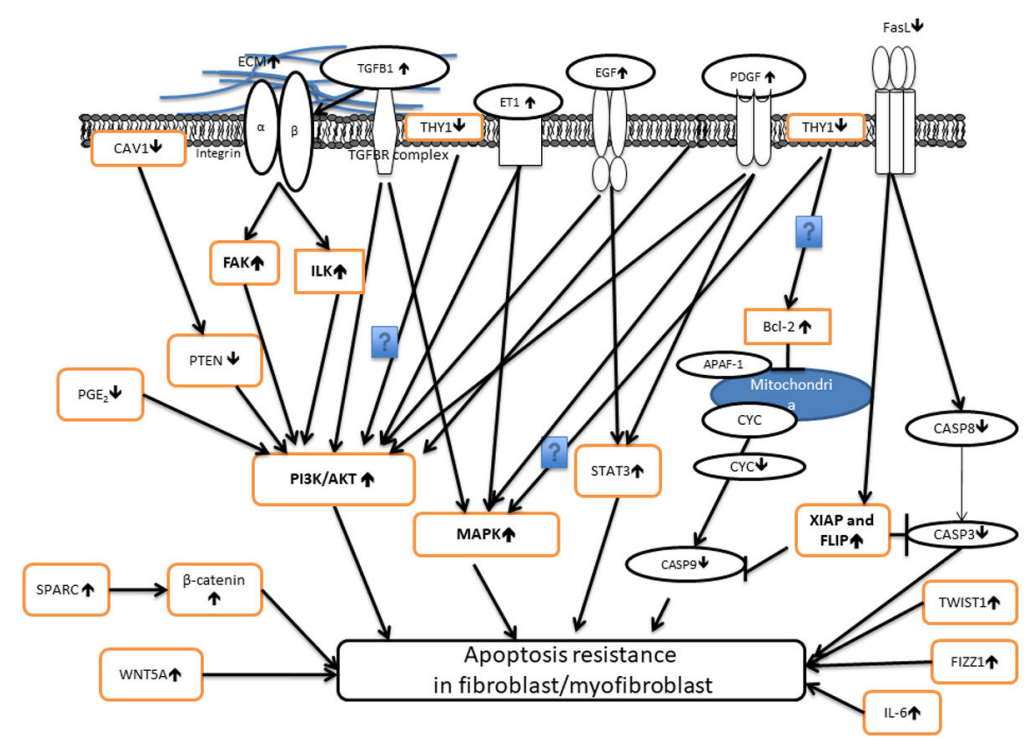

Fig. 3.

Network of selected factors influencing myofibroblast survival/resistance to apoptosis. Several integrin-activated signaling pathways are involved in myofibroblast survival. The prominent role of the AKT survival pathway is demonstrated by the convergence of multiple pathways. Recent results showing the role of WNT/ $\beta$ catenin, TWIST1 and FIZZ1 pathways are also illustrated (The question marks indicate where literature or our own studies suggest an effect, but mechanism is unknown. See text of article). Abbreviations: AKT, v-akt murine thymoma viral oncogene homolog; APAF-1, apoptotic peptidase activating factor 1; Bcl-2, B-cell CLL/lymphoma 2; CASP, caspase; CYC, cytochrome c; ECM, extracellular matrix; EGF, epidermal growth factor; ET1, endothelin 1; FAK, PTK2 protein tyrosine kinase 2; FasL, FAS (TNF receptor superfamily, member 6) ligand; FIZZ1, resistin like alpha; FLIP, CASP8 and FADD-like apoptosis regulator; FOXO3, forkhead box O3; IL-6, interleukin 6; ILK, integrin-linked kinase; MAP, mitogen-activated protein; PDGF, platelet-derived growth factor; $\mathrm{PGE}_{2}$, prostaglandin E2; PI3K, phosphoinositide-3-kinase; PTEN, phosphatase and tensin homolog; SPARC, secreted protein, acidic, cysteine-rich (osteonectin); STAT3, signal transducer and activator of transcription 3; TGFBR, TGFB receptor; TGFB, transforming growth factor, beta; THY1, Thy-1 cell surface antigen; TWIST1, twist homolog 1 (Drosophila); WNT5A, wingless-type MMTV integration site family, member 5A; XIAP, Xlinked inhibitor of apoptosis 


\section{Table 1}

Key mediators of myofibroblast differentiation

\begin{tabular}{|c|c|c|}
\hline Mediator & Potential role or mechanism & Key reference \\
\hline \multicolumn{3}{|c|}{ Cytokines and growth factors } \\
\hline BMP7 & suppression of EMT and EndMT; upregulating ID2 and suppressing liver fibrosis & (Ref. $10,11,170)$ \\
\hline \multirow{2}{*}{ TGFB1 } & initiating myofibroblast differentiation & (Ref. 37) \\
\hline & driving MSC differentiation in combination with CTGF & (Ref. 34) \\
\hline ET1 & inducing EndMT & (Ref. 21) \\
\hline CCL2 & chemoattractant for fibrocytes & (Ref. 26) \\
\hline SDF-1a (CXCL12) & chemoattractant for fibrocytes & (Ref. 24, 27) \\
\hline PDGFB & chemoattractant for fibrocytes & (Ref. 28) \\
\hline SLC & chemoattractant for fibrocytes & (Ref. 24) \\
\hline CTGF & $\begin{array}{l}\text { initiating myofibroblast differentiation; driving MSC differentiation in combination with } \\
\text { TGFB1 }\end{array}$ & (Ref. 34, 35) \\
\hline PDGF & initiating myofibroblast differentiation & (Ref. 37) \\
\hline TNF-a & initiating myofibroblast differentiation & (Ref. 37) \\
\hline IL-1B & initiating myofibroblast differentiation & (Ref. 37) \\
\hline IL-4 & profibrotic in later stages of fibrosis & (Ref. 43) \\
\hline SOCS1 & decreasing TNF- $a$ expression and lung fibrosis & (Ref. 161) \\
\hline TGFB3 & antagonizing TGFB1 and myofibroblast differentiation & (Ref. 45) \\
\hline \multicolumn{3}{|c|}{$\underline{\text { Extracellular matrix and cell contraction }}$} \\
\hline a $3 \beta 1$ integrin & contributing to EMT through Y654- $\beta$-catenin phosphorylation & (Ref. 13) \\
\hline av $\beta 6$ integrin & mediating TGFB1 release via RhoA activation by PAR-1 & (Ref. 60) \\
\hline av $\beta 5$ integrin & mediating contraction-induced TGFB1 release & (Ref. 62) \\
\hline caveolin 1 & inhibiting fibrosis via PTEN induction (associated with $\beta 1$ integrin) & (Ref. 115) \\
\hline \multirow{5}{*}{ THY1 } & resistance to myofibroblast differentiation & (Ref. 44) \\
\hline & decreasing collagen matrix contraction and expression of myogenic markers & (Ref. 52) \\
\hline & inhibiting contraction-induced TGFB 1 release via av $\beta 5$ integrin binding & (Ref. 54) \\
\hline & increasing myofibroblast differentiation in myometrial and orbital fibroblasts & (Ref. 55) \\
\hline & $\begin{array}{l}\text { inhibiting PPAR } \gamma \text {-mediated lipofibroblast differentiation of myometrial and orbital } \\
\text { fibroblasts }\end{array}$ & (Ref. 55) \\
\hline MMP2 & mediating fibroblast migration & (Ref. 28) \\
\hline MMP7 & releasing active TGFB1 & (Ref. 171) \\
\hline MMP8 & mediating fibroblast migration & (Ref. 28) \\
\hline MMP9 & mediating fibroblast migration & (Ref. 28) \\
\hline MMP14 & releasing active TGFB1 & (Ref. 66) \\
\hline TSP-1 & mediating TGFB1 release by binding LAP & (Ref. 44, 61) \\
\hline RAC1 & driving myofibroblast differentiation of HSCs via $\mathrm{HH}$ signaling & (Ref. 65) \\
\hline syndecan 4 & mediating antifibrotic effect of CXCL10; increasing THY1-induced RhoA activation & (Ref. 72, 73) \\
\hline DIA & mediating force-induced myofibroblast differentiation via MRTF-A and SRF & (Ref. 67) \\
\hline \multicolumn{3}{|c|}{ Oxidative stress, cell metabolism } \\
\hline
\end{tabular}

Expert Rev Mol Med. Author manuscript; available in PMC 2017 November 07. 


\begin{tabular}{|l|l|l|}
\hline Mediator & Potential role or mechanism & Key reference \\
\hline NOX4 & driving fibrosis through Smad2/3 activation & (Ref. 77, 78) \\
\hline JUND inactivation (hypoxia) & myofibroblast differentiation via HIF-1a, ERK1/2 and CXCL12 activation & (Ref. 79) \\
\hline SOD & $\begin{array}{l}\text { decreasing lung fibrosis and vascular remodeling via inhibiting TGFB and EGR1 } \\
\text { activation }\end{array}$ & (Ref. 80) \\
\hline PPAR $\gamma$ agonists & $\begin{array}{l}\text { inhibiting lung fibrosis and myofibroblast differentiation in a partially PPAR } \gamma- \\
\text { independent manner }\end{array}$ & (Ref. 81, 82) \\
\hline & \multicolumn{1}{|c|}{ WNT pathway } & (Ref. 89) \\
\hline SPARC & $\begin{array}{l}\text { activating Wnt signaling through AKT and inhibiting plasminogen-induced apoptosis in } \\
\text { IPF fibroblasts }\end{array}$ & (Ref. 90) \\
\hline Dickkopf 1 (WNT antagonist) & inhibiting renal fibrosis &
\end{tabular}

Abbreviations: BMP7, bone morphogenetic protein 7; CCL2, chemokine (C-C motif) ligand 2; CTGF, connective tissue growth factor; CXCL, chemokine (C-X-C motif) ligand; DIA, diaphanous homolog 1 (Drosophila); EGR1, early growth response 1; EMT, epithelial to mesenchymal transition; EndMT, endothelial-mesenchymal transition; ERK1/2, mitogen-activated protein kinase 1/2; ET1, endothelin 1; HH, Hedgehog; HIF-1a, hypoxia inducible factor, alpha subunit; HSC, hepatic stellate cell; ID2, inhibitor of DNA binding 2; IL, interleukin; JUND, jun D protooncogene; LAP, latency-associated peptide; MMP, matrix metallopeptidase; MRTF-A, myocardin-related transcription factor A; MSC, mesenchymal stem cell; NOX4, NADPH oxidase 4; PAR-1, coagulation factor II (thrombin) receptor; PDGFB, platelet-derived growth factor beta polypeptide; PPAR $\gamma$, peroxisome proliferator-activated receptor gamma; PTEN, phosphatase and tensin homolog; RAC1, ras-related C3 botulinum toxin substrate 1; RhoA, ras homolog gene family, member A; SLC, secondary lymphoid tissue chemokine; SMAD2/3, SMAD family member 2/3; SOCS1, suppressor of cytokine signaling 1; SOD, superoxide dismutase; SPARC, secreted protein, acidic, cysteine-rich (osteonectin); SRF, serum response factor; TGFB, transforming growth factor, beta; THY1, Thy-1 cell surface antigen; TNF, tumor necrosis factor; TSP-1, thrombospondin 1; WNT, wingless-type MMTV integration site family 
Table 2

Key mediators of fibroblasts/myofibroblast survival/apoptosis

\begin{tabular}{|c|c|c|}
\hline Mediator & Potential role or mechanism & Key reference \\
\hline \multicolumn{3}{|c|}{ Prosurvival factors: Growth factors and cytokines } \\
\hline TGFB & Activation of prosurvival signaling pathways: PI3K/AKT, ILK/FAK and p38 MAPK pathway & (Ref. 144, 151) \\
\hline PDGF & Activation of prosurvival signaling pathways: PI3K/AKT pathway, MAP Kinases and STAT3 & (Ref. 137, 138) \\
\hline EGF & Activation of prosurvival signaling pathways: PI3K/AKT pathway, MAP Kinases and STAT3 & (Ref. 137, 138) \\
\hline IL-6 & $\begin{array}{l}\text { Upregulation of Bcl-2 in normal fibroblast and BAX in fibroblasts from IPF via STAT3-dependent } \\
\text { mechanism. }\end{array}$ & (Ref. 140) \\
\hline \multicolumn{3}{|c|}{$\underline{\text { Anti-apoptotic proteins }}$} \\
\hline XIAP and FLIP & Inhibiting the activation of caspases & (Ref. 103, 132) \\
\hline Bcl-2 & Inhibiting of mitochondria-mediated apoptosis pathway & (Ref. 133) \\
\hline \multicolumn{3}{|c|}{$\underline{\text { Soluble fibrotic peptides and mediators }}$} \\
\hline FasL/FAS & Increasing XIAP and FLIP proteins & (Ref. 132) \\
\hline ET1 & Activation of prosurvival signaling pathways: PI3K/AKT and p38 MAPK pathway & (Ref. 144) \\
\hline $\mathrm{PGE}_{2}$ & $\begin{array}{l}\text { Regulation of the prosurvival protein kinase (AKT), activation of caspase } 8 \text { and } 9 \text {, increasing the } \\
\text { expression of FAS }\end{array}$ & (Ref. 116) \\
\hline \multicolumn{3}{|c|}{ WNT signaling pathway } \\
\hline SPARC & Activation of AKT, leading to the inhibition of GSK3B and activation of $\beta$-catenin & (Ref. 89) \\
\hline WNT5A & Increasing fibronectin and a 5 integrin & (Ref. 114) \\
\hline$\beta$-catenin & Increasing expression of PAI- 1 and ACTA2 & (Ref. 89) \\
\hline \multicolumn{3}{|c|}{ Anti-apoptosis signaling pathways } \\
\hline AKT & Suppressing of FOXO3 function and increasing cellular expression of XIAP. & (Ref. 172) \\
\hline Ligation of $\beta 1$ integrin & Up-regulation of PI3K/AKT pathway and modulation of ILK/FAK activity & (Ref. 152) \\
\hline \multicolumn{3}{|c|}{ Other mediators } \\
\hline PTEN & Regulation of of AKT activity & (Ref. 143) \\
\hline CAV1 & $\begin{array}{l}\text { Regulation of membrane-associated PTEN expression, AKT activity and TGFB/SMAD signal } \\
\text { transduction via JNK pathway. }\end{array}$ & (Ref. 115, 173) \\
\hline TWIST1 & Shaping their responsiveness to growth factor (TGFB, PDGF and FGF2) stimulation & (Ref. 146) \\
\hline FIZZ1 & activation of ERK and inhibition of caspase 3 and 8 & (Ref. 145) \\
\hline
\end{tabular}

Abbreviations: ACTA2, actin, alpha 2, smooth muscle; AKT, v-akt murine thymoma viral oncogene homolog; BAX, BCL2-associated X protein; Bcl-2, B-cell CLL/lymphoma 2; BIM, BCL2-like 11; CAV1, caveolin 1; EGF, epidermal growth factor; ERK, mitogen-activated protein kinase; ET1, endothelin 1; FAK, PTK2 protein tyrosine kinase 2; FAS, Fas (TNF receptor superfamily, member 6); FasL, Fas ligand; FGF2, fibroblast growth factor 2 (basic); FIZZ1, resistin like alpha; FLIP, CASP8 and FADD-like apoptosis regulator; FOXO3, forkhead box O3; GSK3B, glycogen synthase kinase 3 beta; IL, interleukin; ILK, integrin-linked kinase; IPF, idiopathic pulmonary fibrosis; JNK, JUN N-terminal kinase; MAP, mitogen-activated protein; MAPK, MAP kinase; PDGF, platelet -derived growth factor; PGE2, prostaglandin E2; PAI, plasminogen activator inhibitor type 1; PI3K, phosphoinositide-3-kinase; PTEN, phosphatase and tensin homolog; SMAD, SMAD family member; SPARC, secreted protein acidic and rich in cysteine; STAT3, signal transducer and activator of transcription 3; TGFB, transforming growth factor, beta; TWIST1, twist homolog 1 (Drosophila); WNT, wingless-type MMTV integration site family; XIAP, X-linked inhibitor of apoptosis 